Article original

\title{
L'utilisation des reliefs pariétaux dans la réalisation des signes au Paléolithique supérieur
}

\section{Utilization of parietal relief for realization of signs during Upper Paleolithic}

\author{
Eric Robert* \\ 7, avenue de la République, 92350 Le Plessis-Robinson, France
}

\begin{abstract}
Résumé
La représentation des images du Paléolithique s'est longtemps effectuée de manière à mettre en valeur leurs qualités esthétiques, en ignorant les caractéristiques particulières de leurs supports naturels. L'architecture des grottes ornées, à plusieurs niveaux, participe pourtant pleinement à l'élaboration des dispositifs pariétaux. Eléments omniprésents de ces dispositifs, les signes occupent une place déterminante au sein de cette architecture. L'analyse des relations entre les signes et leurs supports directs permet de mettre en lumière différents modes d'utilisation des reliefs pariétaux, intégration, recherche des volumes et cadrage. Une étude de terrain menée sur un échantillon de 692 signes, répartis dans l'espace paléolithique franco-cantabrique, fait ainsi apparaître la diversité des choix graphiques des artistes face à la paroi.
\end{abstract}

(C) 2007 Elsevier Masson SAS. Tous droits réservés.

\begin{abstract}
For a long time, the images of the Upper Paleolithic were represented in a way that emphasized their aesthetic qualities and ignored the specific characteristics of their natural supports. It is more damageable as the architecture of the decorated caves plays a real part, at different levels, in the elaboration of the parietal devices. As omnipresent elements of these devices, the signs play a determining part in this architecture. Analysing the links between the signs and their direct supports brings to light the various ways in which parietal devices can be used and also the integration and research for the volumes and frame. A study led
\end{abstract}

\footnotetext{
* Laboratoires de rattachement : USM 103 du Muséum National d'Histoire Naturelle, UMR 7041 Arscan, équipe Ethnologie préhistorique.

Adresse e-mail : eric.robert15@wanadoo.fr.
} 
inside the caves on a sample of 692 signs, distributed in the franco-cantabric paleolithic space, makes apparent the variety of the graphic choices left to the artists standing in front of the wall.

(C) 2007 Elsevier Masson SAS. Tous droits réservés.

Mots clés : Signes ; Reliefs pariétaux ; Support naturel ; Topographie des grottes ; Paléolithique supérieur ; Espace franco-cantabrique

Keywords: Signs; Parietal relief; Natural support; Caves topography; Upper Paleolithic; Franco-cantabric area

La représentation des images du Paléolithique s'est le plus souvent effectuée de manière à mettre en valeur leurs qualités esthétiques, à individualiser les plus exceptionnelles d'entre elles.

Toutes ces démarches individualisent ainsi des figures, rarement des signes, donnant une vision décontextualisée de l'art des Homo sapiens.

Pourtant, le contexte des représentations de la préhistoire européenne, en grottes, constitue un élément structurellement différent des autres créations graphiques observables dans le monde et désignés sous le terme d'art « rupestre » (sur roches).

Les artistes du Paléolithique ont, en effet, régulièrement investi ces cavernes, milieu souterrain d'accès et de circulation souvent mal aisés pour y réaliser des représentations animales, humaines, mais aussi de nombreux tracés abstraits, de forme répétitive : les signes. Omniprésents dans l'espace européen, ils possèdent un éventail morphologique très varié, des formes les plus simples (un point ou un trait isolé) aux plus complexes.

Les grottes présentent des profils topographiques et morphologiques diversifiés dont la mise à profit a été progressivement mise en évidence au fur et à mesure de l'avancée de la recherche, d'abord dans leur dimension topographique, avec les travaux de André Leroi-Gourhan et plus récemment à toutes les échelles de leur architecture naturelle.

Eléments clés des dispositifs pariétaux, les signes occupent une place de choix au sein de cette architecture. Bien que souvent considérés comme des éléments secondaires, analysés dans leur rapport aux animaux sous un aspect complémentaire, les signes semblent manifestement avoir fait l'objet de choix particuliers dans leur disposition dans la grotte. Qu'en est-il de leur disposition sur la paroi ?

La lecture des relations entre les signes et leurs supports, et de l'utilisation éventuelle de certains reliefs, doit nous éclairer sur la manière dont les artistes ont mis à profit le support naturel, et par-là même, restituer une part de la démarche graphique des artistes.

\section{La prise en compte progressive du support}

L'importance du contexte naturel n'a pas toujours été prise en compte à sa juste mesure.

À la découverte des premiers témoignages graphiques, c'est l'aspect esthétique des représentations qui a le plus été souligné, même si la forme particulière du plafond d'Altamira et l'inscription des bisons au sein des bosses de ce plafond faisait l'objet d'un constat original de la part de l'inventeur (Sanz de Santuola, 1880).

Celui-ci y voyait un témoignage de "l'instinct artistique ${ }^{1}$ des auteurs de ces peintures polychromes, qu'il rapportait à la période du Paléolithique. On peut également souligner la

\footnotetext{
${ }^{1}$ «Merice también notarse que une gran parte de las figuras están colocadas de manera que las protubancieras convexas de la bóveda están aprovechandas de modo que no perjudiquen en conjunto de aquellas, todo lo que demuestra que su autor no carecía de instinto artístico » (Sanz de Santuola, 1880 : p. 17).
} 
qualité certaine des premières esquisses de relevé qu'il a réalisées. C'est le cas des relevés de plusieurs séries de lignes qui s'avèrent être des signes quadrangulaires, sur lesquels sont inscrites les limites de paroi et les fissures dont plusieurs constituent manifestement un cadre à ces signes.

La forme du grand plafond d'Altamira n'a le plus souvent été soulignée que pour son originalité, alors même que le rôle du support est déterminant dans la forme même des bisons, dont le tracé s'inscrit au sein des bosses et dont le contour suit le rebord naturel de ces bosses.

La représentation qu'en a faite l'abbé Breuil manifeste ainsi, au-delà du talent de dessinateur de leur auteur, d'une démarche particulière dans le relevé, c'est-à-dire dans l'analyse de la figure. Le soin est apporté à la mise en valeur de l'aspect esthétique de la représentation, en l'absence de toute mention du support, alors même que les phénomènes d'utilisations de reliefs étaient identifiés et que leur rôle était décisif.

Une distinction équivalente entre commentaire et relevé s'opère à Font-de-Gaume et aux Combarelles où la place du support est notée dans la lecture des représentations ${ }^{2}$ mais pas dans leurs relevés.

Le renversement des approches qui se produit à partir des années 1950 va construire un mouvement conduisant progressivement à l'intégration du contexte morphologique des grottes dans les recherches en art pariétal.

Max Raphaël, le premier, oriente vers une prise en compte globale du contexte naturel de l'art paléolithique. Selon lui, « chaque caverne possède sa propre distribution topographique des documents artistiques » (Raphaël, 1986 : p. 127).

André Leroi-Gourhan concrétise cette dimension topographique en soulignant la dimension monumentale de cet $\operatorname{art}^{3}$. La sélection, par les hommes du Paléolithique, de certains espaces des milieux souterrains parcourus, témoigne de la « liberté de création de ces artistes » (LeroiGourhan, 1972).

Cette prise en compte de la dimension topographique des réseaux ornés se traduit par la définition d'un schéma de répartition des représentations, aussi bien figuratives que géométriques, selon certaines zones de la grotte, des galeries ou même au sein des panneaux. Dans tous les cas se distinguent essentiellement les espaces centraux des espaces périphériques ou extrémités des secteurs, où les thèmes s'inscrivent différemment. Cela a notamment permis à André Leroi-Gourhan de définir des groupes de signes aussi bien par leur distribution topographique que par leurs caractéristiques morphologiques.

Toutefois, comme on peut le constater à la lecture de certains dispositifs pariétaux, il n'y a pas de mode de répartition uniforme ; notamment pour les signes. Les «normes architecturales » (Vialou, 2004a), variables des cavités, ne permettent pas d'enfermer l'ensemble de l'art paléolithique dans un schéma uniforme. Comme André Leroi-Gourhan le reconnaissait, « la caverne offre un cas particulièrement impropre à la systématisation architecturale de la décoration »(Leroi-Gourhan, 1966 : p. 46). D’une grotte aux multiples ramifications comme Rouffignac à des grottes-couloirs comme les Combarelles ou Marsoulas, on se trouve face à des organisations topographiques variables, qui mettent à profit de manière différente les particularités des réseaux karstiques.

\footnotetext{
2 «Quelquefois, l'artiste a profité des saillies de la pierre pour accentuer certaines parties de l'animal » (Capitan et Breuil, 1901 : p. 494).

3 «L'appropriation de l'espace souterrain n'est pas liée à la fantaisie spontanée de quelques chasseurs : [...] leur liberté de création n'était probablement pas très différente de celle qui, dans les grandes civilisations, régnait durant les périodes de grande décoration monumentale »(Leroi-Gourhan, 1972 : p. 409).
} 
Une étude plus récente, basée sur une lecture des grottes en panneaux classés selon leur contenu et leur position, a tenté de définir un " programme iconographique » à partir de l'organisation spatiale des décors pariétaux (Gonzalez, 2001). Le découpage en panneaux, toutefois, pose un problème quant à sa définition, tout comme leur hiérarchisation et la répartition préférentielle envisagée de certains décors sur certaines surfaces (Lorblanchet, 1993).

L'exploitation par les artistes de leur milieu s'est aussi concrétisée au niveau des parois. Le constat de l'utilisation des reliefs est d'ailleurs effectué par la plupart des observateurs de l'art paléolithique. Les rares exceptions (Ucko et Rosenfeld, 1966) témoignent surtout d'une méconnaissance des réalités pariétales ou d'une mauvaise interprétation de la fréquence de ces utilisations.

L'utilisation des volumes pariétaux est même considérée comme une caractéristique de l'art paléolithique. Annette Laming-Emperaire met ainsi en avant que «le modelé de la paroi ait guidé la main et l'imagination des artistes quaternaires » (Laming-Emperaire, 1962 : p. 26). Elle cite l'exemple des mammouths d'Arcy sur Cure dont les pattes et les trompes s'inscrivent dans les volumes de la paroi.

Toutefois, cette lecture limite cette utilisation des reliefs naturels à des inspirations ponctuelles. On se situe ici dans l'instantané ; c'est le relief qui fait naître l'image dans la tête de l'artiste et qui conduit ce dernier à la réalisation formelle.

La question d'une utilisation contrainte ou d'un opportunisme de la démarche, se confronte à la diversité des exemples constatés. Les signes, à leur échelle, permettent d'apporter des réponses à cette interrogation. La portée du phénomène reste dès lors à appréhender.

La prise en compte de toutes les utilisations de reliefs s'avère donc déterminante. L'application de nouvelles méthodologies de relevés participe à cette démarche. Les travaux du docteur Pales occupent une place prépondérante dans cette évolution.

Son analyse des plaques gravées de la Marche (Vienne) tient, en effet, compte du support et de ses irrégularités à travers la restitution dans les relevés de volumes en surfaces, afin de reconstituer les étapes suivies par le graveur. Cette restitution le conduit à envisager deux démarches possibles : «Dans l'une, l'auteur a pu rechercher le support qui convenait au sujet, dont il portait en lui l'image avec l'intention de la reproduire. Dans l'autre, c'est la forme même de la pierre qui a pu lui suggérer de graver de manière électorale une certaine image parmi toutes celles dont son esprit et sa mémoire visuelle s'étaient meublés » (Pales, 1969 : p. 31).

On le voit, il ne s'agit plus seulement de proposer une démarche opportuniste, mais bel et bien d'envisager une action réfléchie et une véritable recherche des supports, qui ne sont pas nécessairement les plus aisés.

En effet, à la Marche, « les graveurs ont beaucoup plus souvent opéré sur des surfaces accidentées que sur des surfaces planes, alors qu'ils avaient la liberté du choix » (Pales, 1969 : p. 32). Ce constat effectué sur des objets mobiliers pourrait s'étendre aussi aux parois des cavernes, où le plus souvent, les surfaces choisies par les artistes ne sont pas les plus régulières.

L'intégration des reliefs dans les relevés s'est traduite depuis dans l'analyse des représentations pariétales. Sainte-Eulalie (Lot) (Lorblanchet, 1973), Villars (Dordogne) (Delluc et Delluc, 1974) ou l'Aldène (Hérault) (Vialou, 1979) sont les premières à avoir été l'objet d'une prise en compte systématique du support des représentations.

Les éléments de contexte sont parfois même codifiés (Delluc et al., 1986), illustrant une des différentes méthodes mises au point et aujourd'hui utilisées pour la restitution des volumes pariétaux. Cette restitution d'images en trois dimensions sur des surfaces en deux dimensions constitue du reste un des principaux problèmes de l'analyse de l'art pariétal. 
Les solutions graphiques employées se sont diversifiées avec l'arrivée de nouveaux outils, notamment informatiques, et de nouvelles méthodes de relevés, témoignant de la conscience du rôle indubitable joué par le support.

La mise en évidence du rôle du support conduit à les intégrer au-delà de la lecture individualisée des figures, à travers une lecture globale des utilisations de reliefs. Après la caverne participante, ce sont les parois qui sont au centre de ces nouvelles analyses.

Marylise Lejeune pose ainsi le constat suivant : si la configuration de la grotte a pu influencer l'organisation du dispositif pariétal, la structure même de la paroi a pu, elle aussi, intervenir dans l'exécution des figures.

Elle propose une typologie des catégories d'accidents naturels utilisés, "intégrés dans un contexte de traits tels qu'ils peuvent être estimés avoir été volontairement exploités par l'homme du Paléolithique supérieur » (Lejeune, 1985 : p. 16). Cette démarche originale permet de recenser plusieurs catégories d'accidents naturels utilisés, chacune subdivisée en catégories ou types (la typologie des reliefs rocheux, la plus riche, recense pas moins de 19 catégories différentes).

Cette démarche, qui prend pour exemple, seuls les animaux, se caractérise surtout par la lecture d'un mode d'utilisation des reliefs particuliers, celui qui intègre directement les reliefs dans le tracé des représentations. Deux autres aspects sont évoqués et concernent l'usage de volumes ou la recherche d'éléments de cadrage pour la disposition des figures.

D'autres réflexions, plus récentes, ont rappelé l'importance du contexte naturel, en distinguant différents niveaux de lecture de ce contexte dans la grotte (Sauvet et Tosello, 1998), diverses manières d'aborder le support (Pigeaud, 1998).

C'est autour des critères définis par ces chercheurs que nous pouvons identifier les différentes relations entre les signes et leurs supports.

\section{Les signes}

Par signes, il faut se rappeler que l'on entend « l'ensemble des figures abstraites géométriques, de structure élémentaire ou plus élaborée, peintes et/ou gravées, fréquentes dans tous les arts de la préhistoire, parfois, même exclusives » (Paillet, p. 1236 in Vialou, 2004b). Au Paléolithique, même s'ils restent souvent méconnus, ils constituent la part dominante des représentations.

Cette définition peut se compléter par des références faites aux linguistes, les désignant comme des tracés associant à la fois un «signifiant » et un «signifié », un contenant à la forme récurrente et un contenu qui nous échappe mais dont nous présupposons la constance d'une occurrence à l'autre.

Une des principales difficultés se pose au niveau de la délimitation du périmètre des formes que l'on intègre parmi les signes. La constitution de plusieurs typologies a dégagé des formes, types ou clés différents, marquées par les évolutions de la recherche (Sauvet, 1993), autour desquelles de nombreuses extrapolations, pas toujours justifiées, se sont construites.

Les différentes typologies sont liées à des analyses particulières, soit une opposition de deux classes principales, comme l'a progressivement élaboré André Leroi-Gourhan (1958a, 1958b, 1966), soit sur une division en clés basée sur la structure des signes (Sauvet et al., 1977) ou bien encore sur une répartition liée à des caractéristiques régionales, même si elles sont construites sur les logiques différentes (Casado López, 1977 ; Vialou, 1986).

Chacune d'entre elles permet de mettre en évidence la diversité des formes présentes sur les parois de l'espace paléolithique franco-cantabrique. Toutes présentent aussi, à notre sens, des similitudes autour de quelques familles dominantes, sur lesquelles nous nous sommes appuyés pour réaliser la classification indispensable à toute étude sur les signes. 
Nous identifions une dichotomie principale entre tous les types, conduisant à distinguer deux familles principales, distinction proche de celle élaborée pour les signes ariégeois par Denis Vialou.

La première famille rassemble les formes les plus simples que l'on peut classer sous deux types principaux : les ponctuations et les traits. La seconde famille rassemble des formes plus élaborées, à caractère géométrique, très diversifiée (Fig. 1).

Les ponctuations et les traits peuvent être découpés en plusieurs sous-catégories selon deux axes principaux : la taille et le nombre.

À titre d'exemple, parmi les ponctuations, on distingue les petites, plus fréquentes, réalisées au doigt ou au pinceau, des ponctuations larges, le plus souvent réalisées au soufflé ou au tamponné. L'aspect technique est ici primordial dans la différenciation et revêt un lien particulier avec la forme du signe.

Quelles que soient leur taille et leur technique de réalisation, les ponctuations peuvent aussi se distinguer selon leur organisation : seules, par paire, en alignement, en cercle, en groupe... La multiplicité de ces modes d'agencement témoigne de la grande variété de formes que l'on rencontre dans les grottes du Paléolithique supérieur et notamment au sein du Magdalénien ariégeois.

Les traits offrent une moins grande variété de sous-types. On peut toutefois, opérer également une distinction par la taille ; traits courts et traits longs distinguant deux gestes différents des artistes. Il existe également plusieurs modes d'organisation, entre trait seul, par paire ou alignés en série(s).

Ponctuations et traits se distinguent des autres signes car ils présentent une variété de composition, alors que les types et les formes qui composent la deuxième famille se distinguent, eux, par une variété dans leur conformation interne.

Parmi ces autres catégories, certaines sont présentes dans tout l'éventail géographique et chrono culturel du Paléolithique supérieur, d'autres uniquement dans certaines régions (le plus souvent spécifiques d'une même période), d'autres encore spécifiques d'une seule grotte.

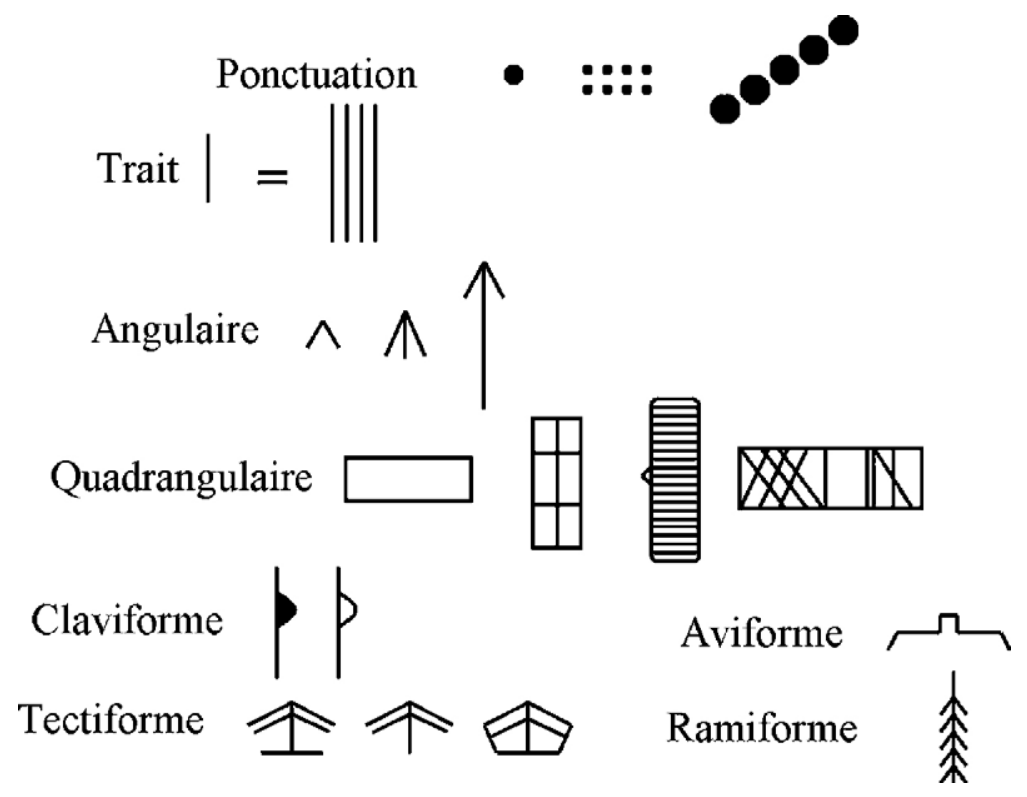

Fig. 1. Exemples de signes présents dans les grottes de l'espace franco-cantabrique.

Fig. 1. Variety of signs distributed inside caves of franco-cantabric area. 
Plutôt que de créer de nouvelles appellations, nous nous sommes efforcés de rassembler, lorsque cela était possible, les catégories des différentes typologies. Nous nous sommes appuyés pour cela sur des divisions géométriques « classiques » (angulaire, cercle et ovale, croix, triangle, quadrangulaires) et sur les types originaux identifiés communément par tous les auteurs (aviformes, claviformes, tectiformes, ramiformes).

Tous ces types, parfois divisés en sous-catégories, correspondent dans la diversité des cas rencontrés dans les cavités du Paléolithique supérieur européen.

Une catégorie de signes se distingue toutefois, que nous n'avons pas pu intégrer dans nos catégories. Ces signes « inclassables » ne sont le plus souvent présents que dans une seule grotte, et couvrent un spectre morphologique assez large.

On y retrouve des tracés associant de manière originale les types les plus simples, comme ce signe de la grotte de Marsoulas (Haute-Garonne, France) qui associe points et traits (Fig. 2) et dont on retrouve un semblable dans la grotte de Bédeilhac.

Mais la plupart des tracés présentent des contours souvent éloignés des types traditionnels et dont la forme générale a pu prêter à des rapprochements tantôt avec des objets (les signes « en cloche »d'El Castillo, (Fig. 3) ; les « fuseaux » des Combarelles, Fig. 4), avec des éléments anatomiques (les «bras » de la grotte de Santian) ou rappelant les animaux, tels que les « serpentiformes » de Rouffignac ou le pseudoaviforme du Portel, distinct de ceux du Quercy ou du Placard (Fig. 5).

À de rares exceptions près, ces tracés apparaissent souvent à plusieurs reprises, voire en séries dans les grottes. Ils manifestent de surcroît une tendance certaine à l'agrégation dans un même secteur de la grotte. C'est le cas dans plusieurs grottes des Cantabres, notamment à la Pasiega, où les signes ovalaires à excroissance se concentrent dans le fond de la Pasiega A où ils partagent l'espace avec les signes quadrangulaires, avec lesquels ils présentent des similitudes morphologiques par leur cloisonnement interne ou la présence d'une excroissance.

Au final, la répartition typologique permet de souligner les récurrences morphologiques et de distinguer les signes dans la variété de leur mode de composition (pour les points ou les traits) ou

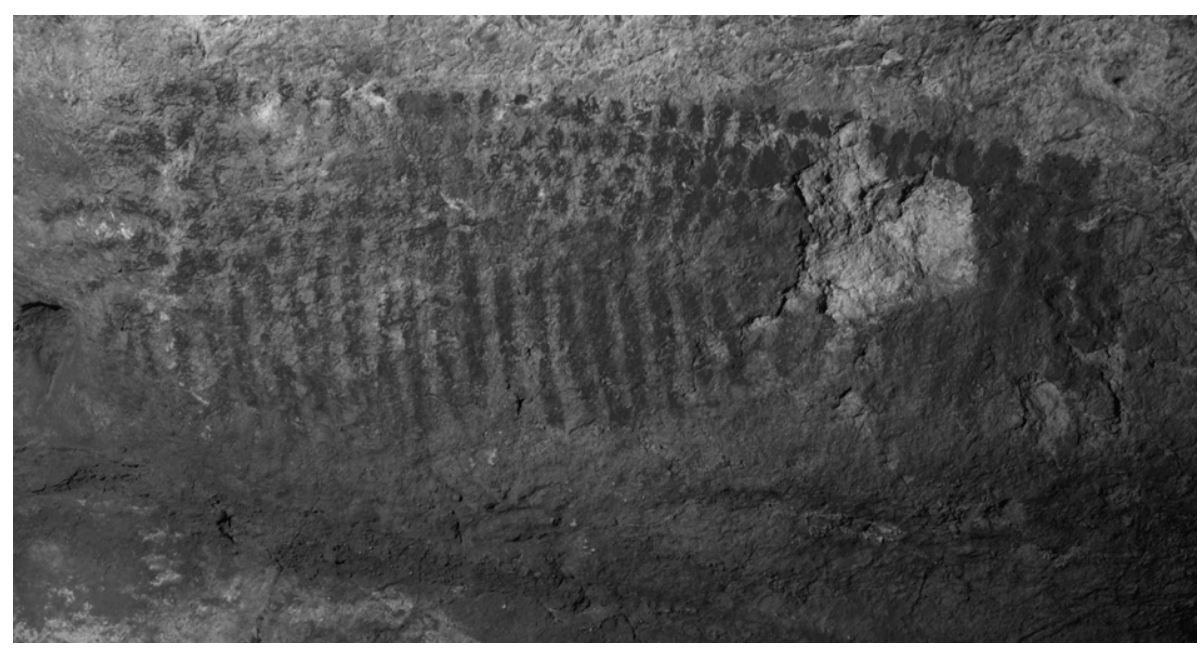

Fig. 2. Signe formé de points et de traits, Marsoulas (Pyrénées) (cliché Carole Fritz et Gilles Tosello).

Fig. 2. Sign constituted by points and lines, Marsoulas (Pyrénées) (photography Carole Fritz and Gilles Tosello). 


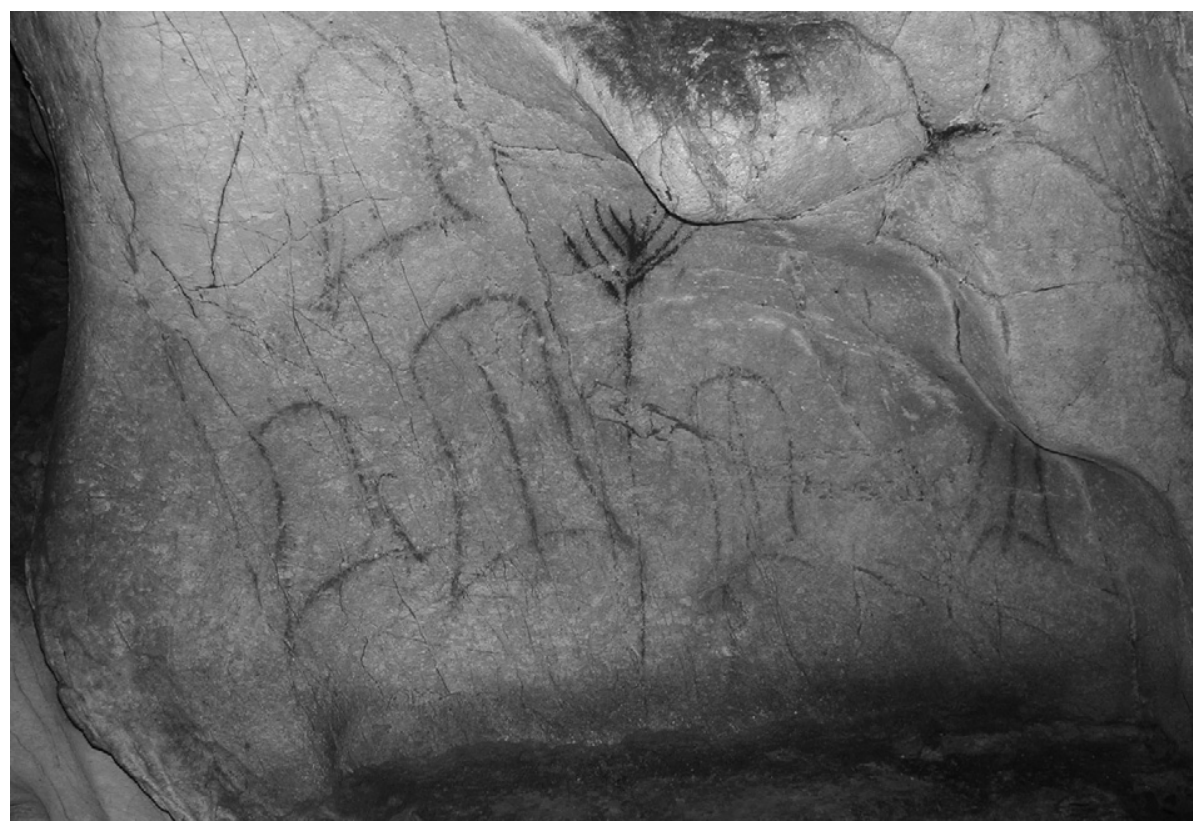

Fig. 3. Signes « en cloches » rouges d'El Castillo (Cantabres) (cliché Eric Robert). Fig. 3. "Bell” red signs, El Castillo (Cantabres) (photography Eric Robert).

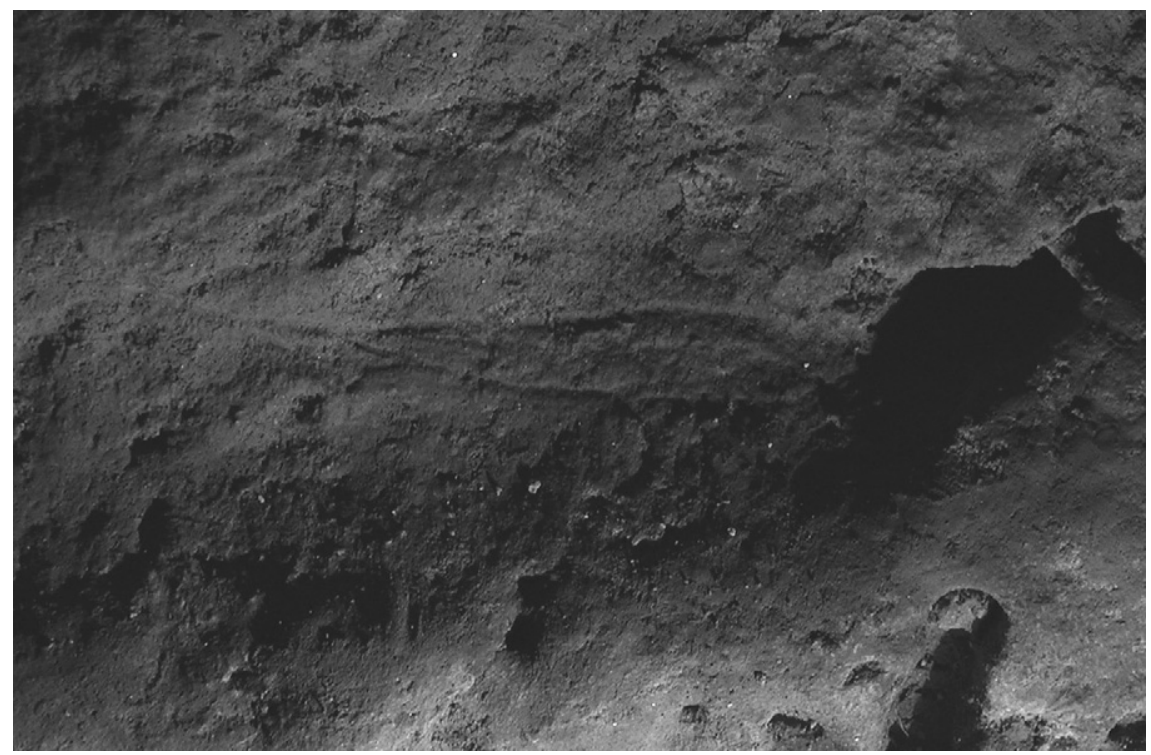

Fig. 4. Signe « en fuseau » gravé des Combarelles (Dordogne) (cliché Eric Robert).

Fig. 4. "Spindle" engraved sign, les Combarelles (Dordogne) (photography Eric Robert). 


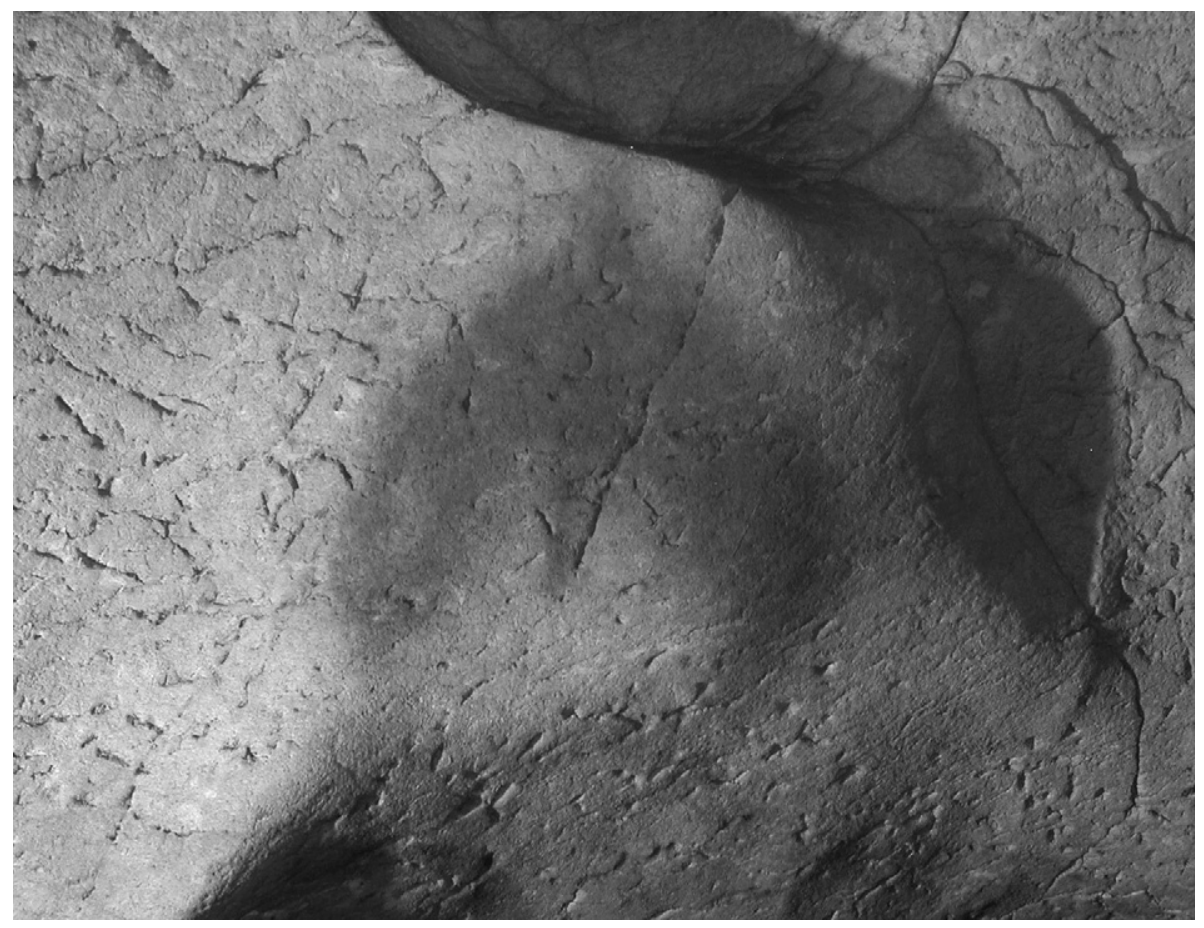

Fig. 5. Signe « en oiseau » rouge du Portel (Pyrénées) (cliché Eric Robert).

Fig. 5. "Bird" red sign, le Portel (Pyrénées) (photography Eric Robert).

de conformation (pour les autres familles de signes). Elle ne répond, toutefois, jamais complètement à la multitude des formes abstraites et de leur constante complexité.

\section{L'analyse du contexte naturel des signes}

Afin d'appréhender la diversité des situations proposées par cette complexité et de vérifier l'investissement accordé par les artistes du Paléolithique supérieur à la réalisation de chacun de leur tracé, il était nécessaire d'intégrer la lecture des utilisations des reliefs pariétaux dans une méthodologie appropriée.

Nous avons, dans le cadre d'une recherche doctorale (Robert, 2006), pris en compte trois catégories d'informations : des informations sur la nature du signe (taille, technique, type, orientation) ; sur le contexte naturel du signe (répartition topographique, forme de la paroi, utilisation des reliefs pariétaux); sur les liaisons graphiques éventuelles du signe, avec d'autres signes ou avec des animaux.

Le croisement de ces catégories d'informations devient ainsi un moyen de mieux appréhender les choix qui ont présidé à la réalisation des signes, des séries abstraites au cœur des dispositifs pariétaux. Il permet surtout de ne pas reproduire à l'inverse une démarche que nous avons critiquée qui s'intéresserait cette fois uniquement au support en négligeant les caractéristiques techniques et le contexte graphique.

L'axe structurant de la démarche se situe dans l'analyse du contexte naturel des signes. Les figures pariétales s'intègrent dans une architecture complexe, « reposant sur une succession de niveaux d'expression emboîtés » (Vialou, 2004a : p. 7). 
Afin de mettre en perspective «l'espace graphique » au sein duquel s'inscrit l'utilisation des reliefs pariétaux, nous allons préalablement décliner les deux niveaux, topographiques et macromorphologiques qui eux-mêmes s'inscrivent dans deux espaces distincts, respectivement, un espace architectural et un espace pariétal.

\subsection{L'organisation topographique}

L'enregistrement de la répartition dans les grottes s'inscrit dans un espace architectural, l'échelle la plus large du contexte naturel.

Nous avons cherché, par l'utilisation de critères généralistes applicables dans toutes les grottes, à observer d'éventuelles organisations particulières des signes, tout en observant la fidélité de leur répartition au modèle défini par André Leroi-Gourhan.

En effet, la question de la répartition topographique des signes est restée limitée à ce schéma uniforme au sein duquel certains types occupent une place définie, au centre ou aux extrémités des galeries, des panneaux ou de l'ensemble de la grotte. Les éléments de ce schéma ne correspondent pas à la réalité de certains dispositifs paléolithiques et non des moindres. Ainsi, les signes quadrangulaires complexes, censés occupés le centre des dispositifs, se trouvent à l'extrémité de la Pasiega A.

D'autres exemples viennent ainsi a contrario du schéma proposé, d'autant plus importants qu'ils concernent des séries importantes de signes.

Un exemple comparatif de deux séries de signes illustre que des types différents peuvent occuper une place semblable dans des contextes karstiques contrastés : les claviformes dans la grotte de Niaux et les ponctuations dans la grotte de Villars.

Ces deux cavités appartiennent à des horizons géographiques différents, leurs dispositifs pariétaux à des cultures différentes, elles aussi (le Magdalénien moyen pour Niaux, la fin du Solutréen ou le Magdalénien ancien pour Villars).

On observe pourtant un phénomène assez similaire, puisque ces deux types de signes se répartissent tout au long du parcours pariétal souterrain, tout en évitant les principaux centres ornés : le panneau principal de la salle des peintures à Villars, la rotonde du Salon-Noir à Niaux.

Les ponctuations de Villars, presque toutes noires, se situent tout au long du réseau karstique, notamment à la fin de galeries, au niveau de passages étroits et conduisent à parler de « jalonnement» (Delluc et Delluc, 1974 : p. 61) (Fig. 6).

Les trois points situés le long du rebord d'un plancher stalagmitique, suspendu au niveau du tournant conduisant vers les salles profondes, illustrent parfaitement cette position particulière des points dans la grotte de Villars (Fig. 7). De surcrôt, pour la majorité d'entre eux, ils se situent préférentiellement sur des surfaces plutôt bosselées, accentuant leur mise en évidence.

Les seuls points présents au milieu du panneau principal de la grotte, situé à la fin du parcours actuel, sont aussi les seuls rouges, (un signe ramiforme est également peint en rouge). Les autres points ne sont par ailleurs, le plus souvent, pas associés à d'autres représentations, particulièrement à des représentations figuratives (deux points noirs sont associés à un bison noir dans la salle des Cierges).

On perçoit donc une corrélation entre emplacement topographique, choix de la surface, technique et association avec les animaux, pour un même type de signe, les points, dans cette cavité. Cette concordance entre différents critères manifeste les choix originaux effectués par les artistes de Villars.

À Niaux, les claviformes se trouvent aussi disposés aux extrémités de plusieurs secteurs de la grotte, notamment à la fin de la galerie d'entrée et au début de la galerie profonde (Fig. 8). Ils sont 


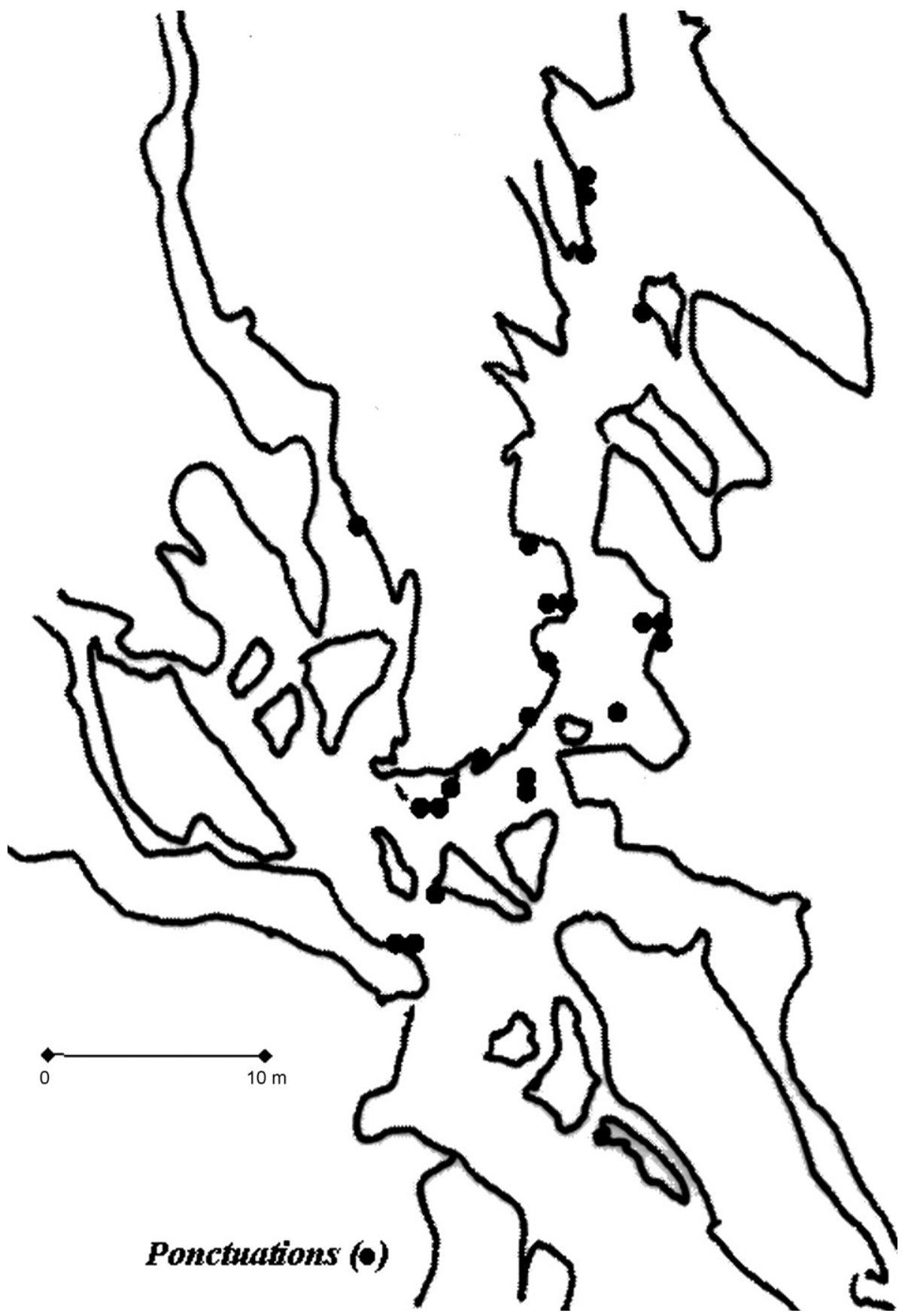

Fig. 6. Plan de répartition des ponctuations de la grotte de Villars (Dordogne).

Fig. 6. Punctuations distribution on draught of Villars cave (Dordogne). 


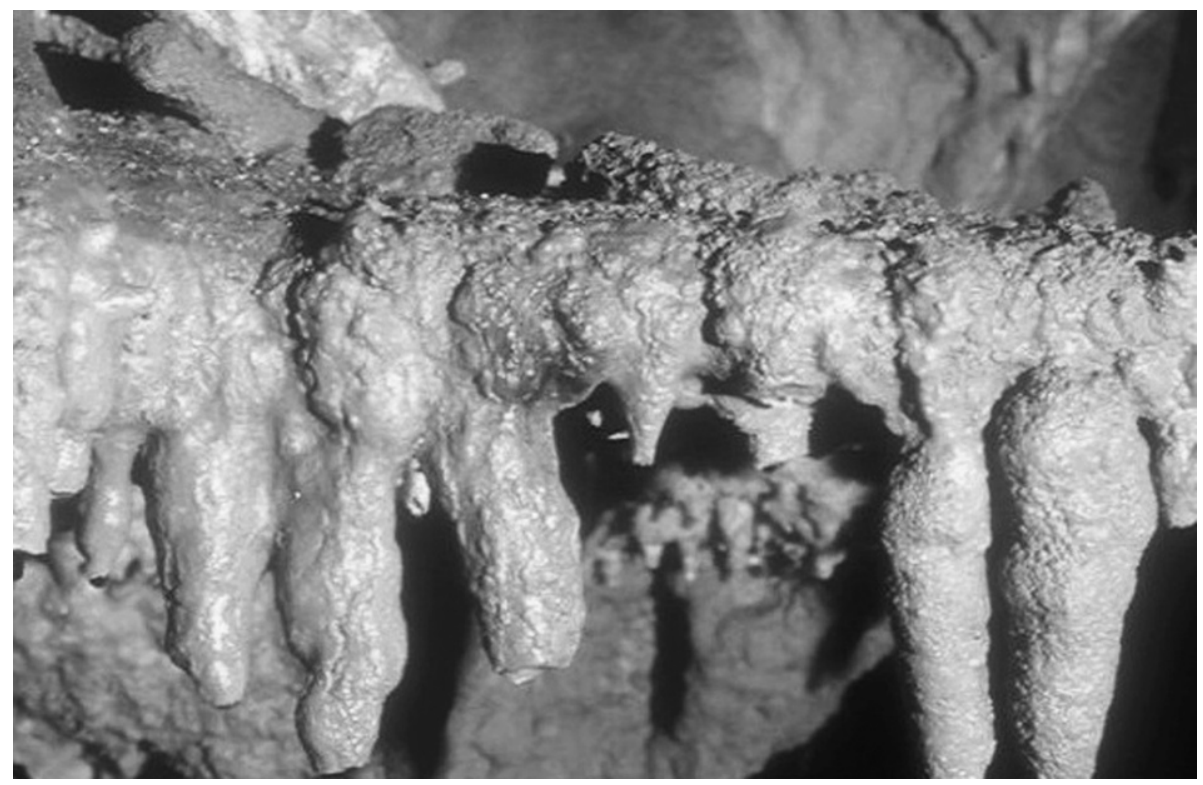

Fig. 7. Ponctuation noire sur un plancher stalagmitique, Villars (Dordogne) (cliché Brigitte et Gilles Delluc). Fig. 7. Black punctuation on a stalagmite hanging floor, Villars (Dordogne) (photography Brigitte and Gilles Delluc).

sur des panneaux comprenant d'autres motifs abstraits, notamment des points, avec lesquels ils forment une association privilégiée, à Niaux mais aussi dans d'autres cavités ariégeoises ou cantabriques.

Ils encadrent le « carrefour » depuis lequel partent les cheminements menant vers la galerie de l'Éboulis, les galeries profondes et le Salon-Noir.

On trouve enfin des claviformes disposés de part et d'autre de la rotonde du Salon-Noir. Ces trois signes évitent soigneusement les principaux panneaux. Parmi eux, un seul est

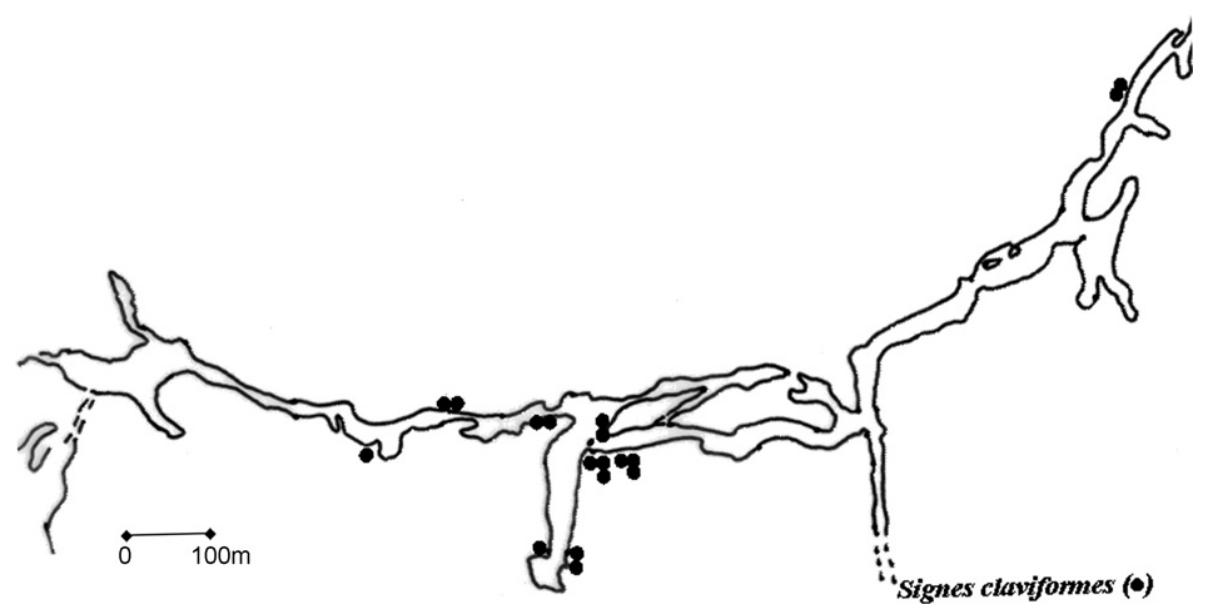

Fig. 8. Plan de répartition des claviformes de la grotte de Niaux (Pyrénées).

Fig. 8. Claviformes distribution on draught of Niaux cave (Pyrénées). 
associé par contact direct avec un animal, un bison superposé au niveau de la croupe à ce claviforme.

Les deux autres sont en face de ce signe, disposés dans une faille, assez large pour constituer un passage étroit. Ils se trouvent à l'écart des panneaux de la rotonde et sont associés à des alignements de points verticaux, parmi les rares signes de ce type présents dans le Salon-Noir. L'orientation de ces signes est par ailleurs liée à une fissure verticale qui court le long de la paroi gauche contre laquelle s'appuie l'un des claviformes.

Les claviformes apparaissent donc ici clairement comme un élément structurant du dispositif pariétal, en étant disposés à des endroits stratégiques du déroulé karstique. Cela est d'autant plus intéressant de souligner que ce mode de répartition et d'organisation n'a semble-t-il pas d'équivalent dans les autres grottes, où ils sont présents, que ce soit en Ariège ou dans les autres régions, où ils ont été mentionnés. On les trouve en effet plus souvent en série, avec des effectifs moins importants, hormis dans les cavernes des Trois-Frères et du Tuc-d'Audoubert où ils se comptent par dizaines, regroupés dans des secteurs ou sur des panneaux spécifiques, plutôt que répartis dans toutes les galeries.

Il est donc possible que les claviformes occupent à Niaux une place tout à fait originale et différente de celle qu'ils occupent dans les autres ensembles contemporains du Magdalénien ariégeois ou cantabrique.

\subsection{La macromorphologie}

Entre l'échelle topographique et l'utilisation des reliefs pariétaux, s'intercale un échelon correspondant à ce que Georges Sauvet et Gilles Tosello ont dénommé comme étant des « macroreliefs » (Sauvet et Tosello en 2000), espace pariétal original dans lequel s'inscrivent une part non négligeable des représentations pariétales, et notamment des signes.

Les critères de sélection de ces reliefs sont basés sur leur aspect monumental : il s'agit notamment de colonnes ou de piliers stalagmitiques, de pendants rocheux, inévitables sur le parcours. S'y associent des espaces beaucoup plus discrets, comme les niches (Fig. 9), les camarins, les renfoncements...

Ces différentes situations témoignent soit d'une recherche de mise en évidence particulière, soit au contraire d'une volonté de dissimulation, voire de protection des œuvres qui y sont représentées.

Ces reliefs accueillent souvent des ensembles ou des séries de signes, comme c'est le cas à Font-de-Gaume, où plusieurs tectiformes occupent un recoin, la salle des petits bisons ou bien à Cougnac où la majorité des aviformes se situent dans un renfoncement, témoignant d'une discrétion que le seul exemple d'aviforme de Pech-Merle ne dément pas en étant situé dans un recoin d'une galerie.

Cette tendance à l'agrégation sur ces macroreliefs est tout à fait remarquable pour les signes quadrangulaires des grottes cantabriques, au sein desquelles « certaines figures ou certains groupes de figures semblent proches ou même placés sur un même type de forme naturelle de la cavité qui, par sa forme ou sa localisation, présente des singularités [...] » (Gonzalez, 2001 : p. 31).

On constate, en effet, aussi bien dans les grottes du Monte Castillo que sont las Chimeneas, el Castillo et la Pasiega, qu'à Altamira, que les signes de type quadrangulaire ont tendance à se situer dans des espaces étroits, occupant un même panneau, une même paroi, parfois sur plusieurs mètres de long.

Une des particularités de ces rassemblements tient dans le fait qu'à plusieurs reprises, ils ont tendance à se faire dans des espaces plutôt discrets. C'est le cas pour le « Recoin-aux- 


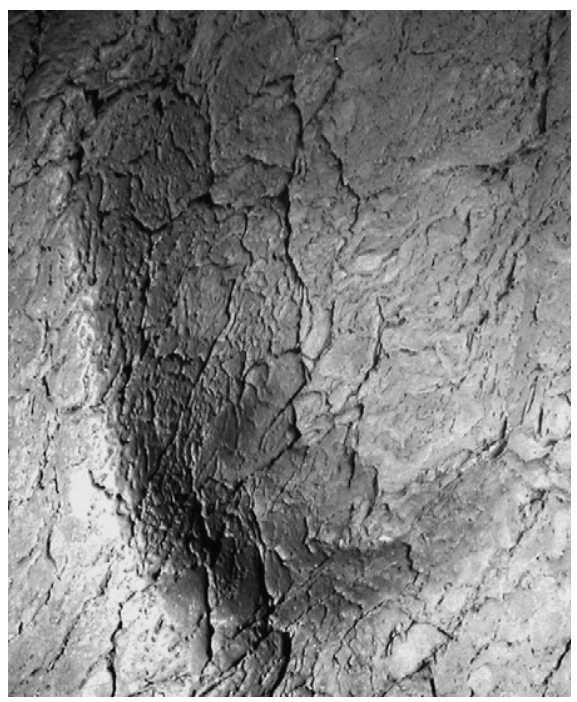

Fig. 9. Ponctuation rouge dans une niche, le Portel (Pyrénées) (cliché Eric Robert). Fig. 9. Red punctuation in a recess, le Portel (Pyrénées) (photography Eric Robert).

Tectiformes » à el Castillo ou pour la diaclase finale de la Pasiega A (Fig. 10) ou bien encore à Altamira.

La grotte de las Chimeneas se démarque des précédentes, puisque les signes quadrangulaires y occupent un fronton rocheux au milieu de la salle principale de la grotte (Fig. 11). Cet emplacement témoigne à l'inverse d'une volonté de mise en évidence et non de dissimulation.

Le fait que les quadrangulaires soient dessinés en noir à las Chimeneas (la peinture rouge est absente dans la grotte, les autres figures sont gravées) alors qu'ils sont rouges voire jaunes (quelques-uns à la Pasiega) dans les deux autres grottes du Monte Castillo, souligne une conjonction entre technique et emplacement qui, par ailleurs, ne s'impose pas comme une règle immuable dans les ensembles du Paléolithique supérieur que nous avons observés.

Alors qu'une même tendance à l'agrégation apparaît ainsi pour les quadrangulaires de la région cantabrique, une dissociation apparaît à la lecture de la nature des supports des représentations abstraites. Cette dissociation pourrait témoigner d'un rôle différent tenu par les quadrangulaires d'une grotte à l'autre, dans les cavités du Monte Castillo.

\section{Les modes d'utilisation de la paroi}

La dernière échelle de lecture correspond au support direct de représentation du signe. Le terme de support nécessite une clarification sur le sens qui lui est accordé. Pour Michel Lorblanchet, le support dans l'art pariétal, « est la paroi rocheuse qui a été modifiée par l'artiste préhistorique » (Lorblanchet, 1993 : p. 9).

Plutôt que de modification, qui signifierait selon nous une transformation de la paroi, «nous préférons parler d'investissement, afin d'intégrer, à la fois l'action technique (peinture ou gravure) à l'origine de la réalisation de la figure et le possible utilisation des reliefs naturels de ce support » (Robert, 2006 : p. 31).

L'observation du support se traduit en détail à l'observation des microreliefs, supports directs des représentations. 


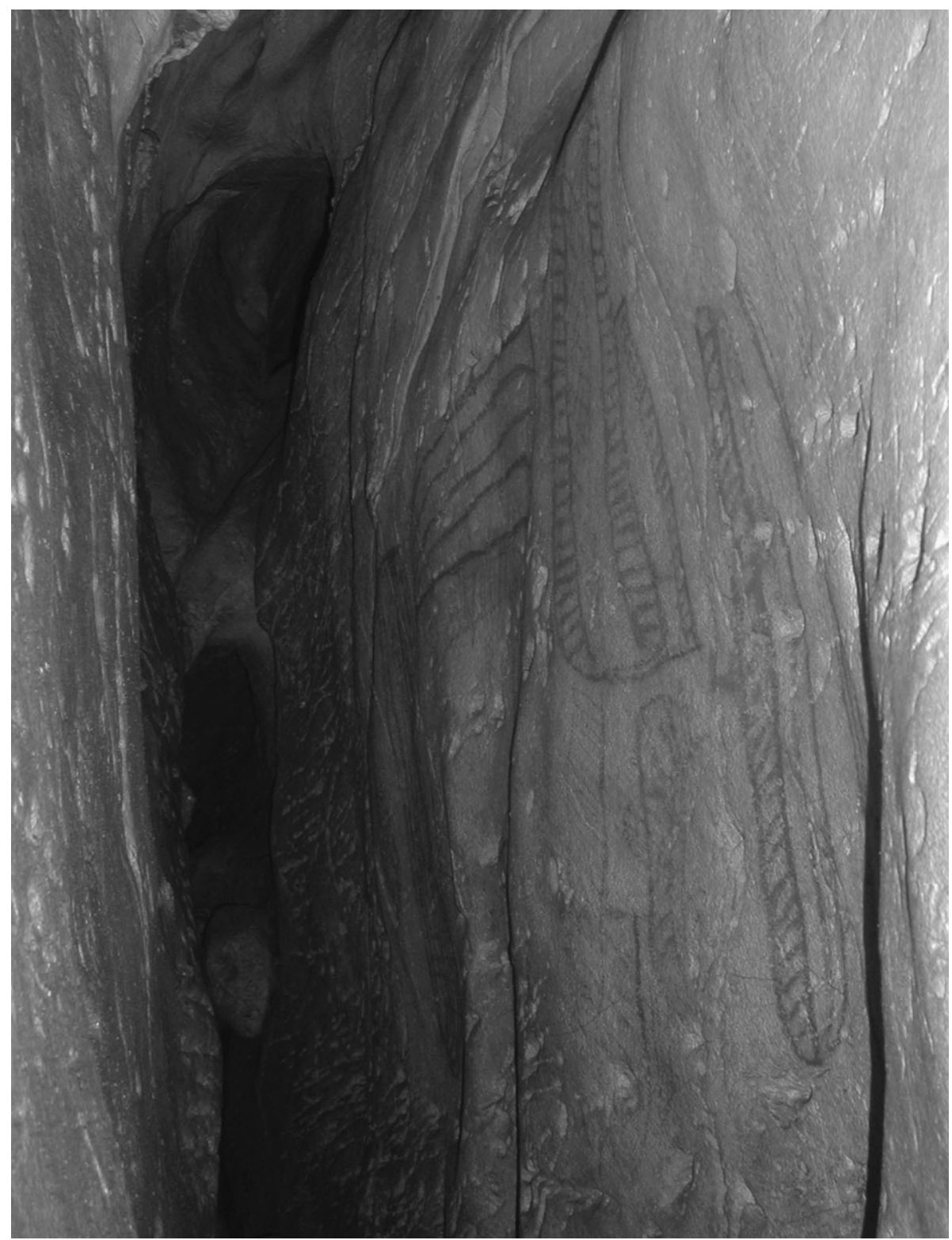

Fig. 10. Quadrangulaires rouges de la diaclase finale de la Pasiega A (Cantabres) (cliché Eric Robert).

Fig. 10. Red quadrangulars disposed on final narrowness, la Pasiega A (Cantabres) (photography Eric Robert).

La lecture de chacune des deux échelles précédentes, topographique et macromorphologique, a révélé leur importance pour la compréhension de la constitution des dispositifs pariétaux.

La lecture des surfaces de réalisation doit éclairer de quelle manière, dans la construction et la disposition de chaque tracé abstrait, au cœur de leur espace graphique, les artistes ont mis à profit les microreliefs des parois. Cette mise à profit correspond à « la sélection d'un champ d'espace 


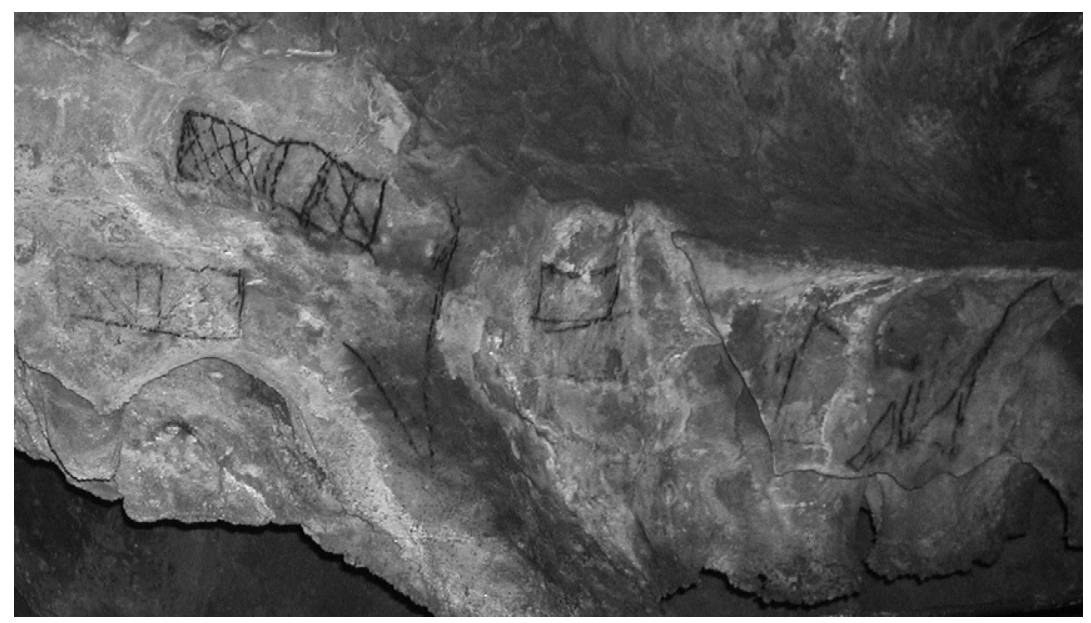

Fig. 11. Quadrangulaires noirs, fronton de la salle centrale de Las Chimeneas (Cantabres) (cliché Eric Robert).

Fig. 11. Black quadrangular, disposed on a fronton of central room, Las Chimeneas (Cantabres) (photography Eric Robert).

sur un support pariétal dont les caractéristiques de formes, de volumes et de situation offrent des possibilités de cadrage et de réalisation d'une œuvre d'art pariétal » (Pigeaud, 1998 : p. 9).

À travers cette définition, on perçoit d'ores et déjà plusieurs manières d'appréhender le support naturel et d'y insérer les représentations, plusieurs modes d'utilisations des reliefs pariétaux.

Le travail réalisé par Marilyse Lejeune a permis une première classification des accidents naturels, qui se concentre toutefois vers un mode particulier, celui de l'utilisation directe de reliefs dans le tracé même de la représentation.

Dans ce mode d'intégration des reliefs au contour du sujet, nous observons littéralement les éléments de la paroi situés dans le corps du tracé. Ce mode d'utilisation « spectaculaire » est le plus fréquemment souligné dans l'observation des figures ; il est celui qui attire le plus de commentaires, mais aussi contre lequel se sont dressées les plus vives critiques.

Le second mode d'utilisation concerne plus particulièrement la recherche des microvolumes de la paroi destinés à une mise en valeur de la surface d'ensemble de la représentation. Il s'agit de reliefs concaves ou convexes, de longueur et de largeur variable, distinguant par exemple, les creux ou les bosses, des saillies ou des crêtes.

Le troisième et dernier mode d'utilisation que l'on peut distinguer correspond quant à lui aux éléments naturels qui participent à la définition d'un « champ-limite » (Otte, 1997 : p. 17). Ces reliefs naturels déterminent un cadrage des signes, cadrage qui peut être partiel ou entier.

Par ces trois modes, nous distinguons toutes les utilisations de reliefs situés dans, sous et autour du signe, au niveau du support direct de la représentation. Elles ont pu être décrites différemment selon les auteurs : on parle notamment d' « utilisation révélée » (Pigeaud, 1998) ou de «substitution d'un relief à un élément constitutif du sujet» (Sauvet et Tosello, 1998 : p. 61) pour désigner l'intégration des reliefs. Mais elles décrivent bien les mêmes phénomènes.

Nous identifions surtout derrière ces modes des gestes différents des artistes, des démarches particulières qui peuvent aussi être complémentaires.

D'autres utilisations ont été distinguées, qui toutefois concernent des cas plus rares et plus problématiques dans leur interprétation. Inadaptées aux signes, nous ne les avons pas reprises. 


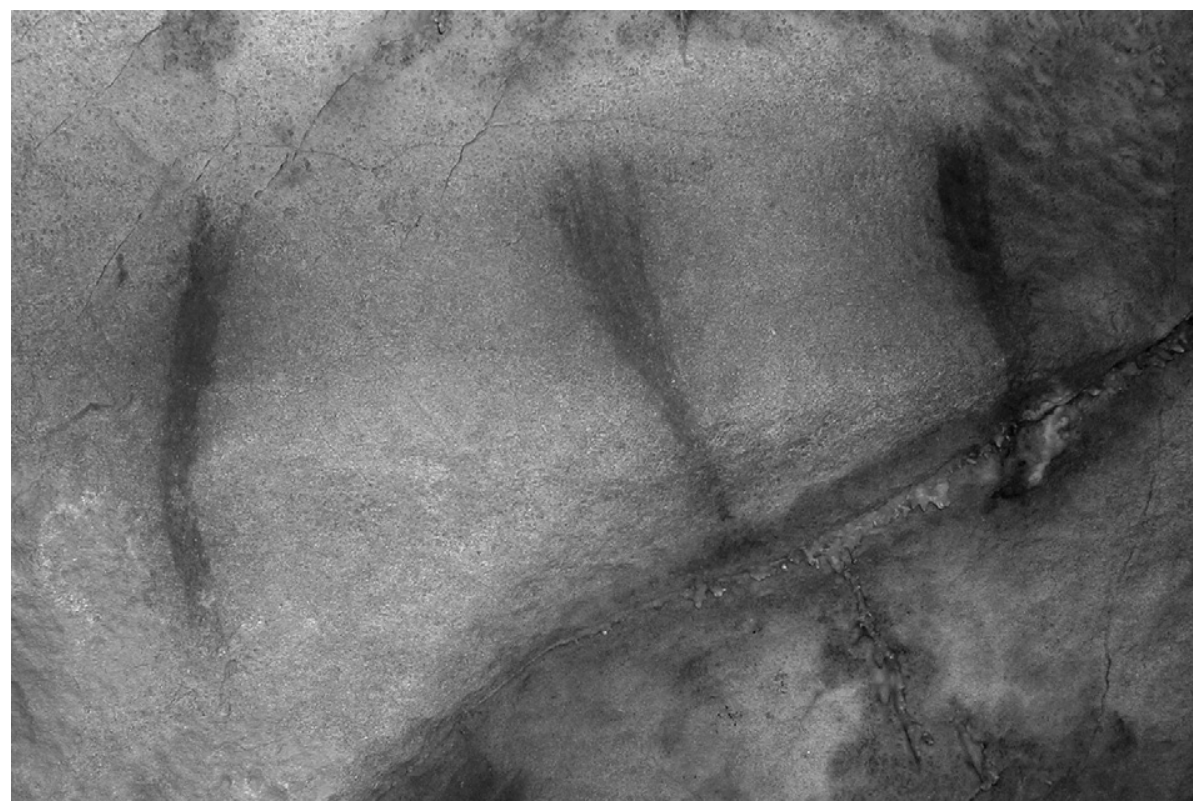

Fig. 12. Signes « en bras » rouges, peints « à rebours » des reliefs, Santian (Cantabres) (cliché Eric Robert).

Fig. 12. "Arm" red signs, painted against the run of the landscape, Santian (Cantabres) (photography Eric Robert).

Une démarche originale apparaît à la lecture de certaines œuvres, la réalisation graphique « à rebours du support », pour laquelle « [...] le Paléolithique a tenu compte des possibilités de son matériau, pour ensuite s'en affranchir » (Pigeaud, $1998:$ p. 12).

Dans ce cas précis, le tracé du signe ou de la figure ne tient aucun compte des irrégularités de la paroi, traversant ainsi les fissures, les alternances de niveau. . (Fig. 12). L'image mentale dans l'esprit de l'artiste est reproduite comme telle sur la paroi. Ces exemples ne sont pas les plus fréquents mais ils illustrent un comportement différent de l'artiste dans sa réalisation graphique face à la paroi.

Ils démontrent aussi la capacité des hommes du Paléolithique à s'affranchir des contraintes naturelles et en négatif, que l'utilisation des volumes pariétaux dans la construction ou la disposition de chaque représentation ne traduit pas une limite technique de l'artiste, mais bel et bien un choix graphique.

La problématique humaine qui transparaît à travers l'ensemble des modes d'utilisation est celle du rapport entre l'artiste et son support, de la relation de « dépendance/maîtrise » de l'homme face à la paroi. Identifier la part contraignante et celle du choix est un des éléments que nous avons essayé de mettre en évidence à la lecture de chaque signe, mais aussi à l'observation de répétitions éventuelles.

\section{Les signes et leurs supports : la méthode d'analyse}

Ces modes d'utilisation ont été intégrés, comme nous l'avons présenté, dans la méthodologie construite sur la comparaison de critères que nous avons mise en place.

L'analyse des supports directs des signes nécessite une condition impérative : l'observation directe de l'emplacement des signes sur les parois. La mention des utilisations de reliefs reste 
trop souvent absente des publications, en particulier pour les signes, pour permettre une approche bibliographique.

L'évolution positive des travaux de recherche ces dernières années, vers une matérialisation graphique des reliefs pariétaux, sous des formes diverses, reste encore insuffisante en terme quantitatif, car elles ne portent que sur quelques grottes.

De surcroît, l'expérience offerte par la visite des cavités reste inégalable, pour restituer réellement la place occupée, aussi bien sur les parois qu'au sein de la grotte, par les signes. Les descriptions des lieux, aussi détaillées soient-elles, ne permettent pas de rendre compte aussi clairement de la situation des signes que la lecture directe des emplacements et des répartitions.

Aussi, nous avons procédé à une étude de terrain qui nous a permis de dresser un premier bilan sur un échantillon de 692 signes répartis dans 26 grottes (Robert, 2006). L'illustration des différentes facettes de mise à profit des supports naturels concernait nécessairement les contextes souterrains les plus variés, aussi bien au niveau de la répartition des territoires, que des cultures ou des milieux karstiques.

L'échantillon des sites et des signes, répond donc à cet objectif de diversité, ainsi qu'aux contraintes naturelles d'accès aux sites ${ }^{4}$. Le nombre des signes analysés dans chaque grotte ne correspond donc pas à la totalité des manifestations graphiques abstraites de chacune des cavités (Tableau 1).

Les sites se répartissent quasi-exclusivement entre Dordogne, Quercy, Ariège et Cantabres ; ces quatre régions concentrent une part importante des grottes ornées au Paléolithique supérieur. Toute la période est du reste couverte, de l'Aurignacien avec les gravures de l'Aldène au Magdalénien, période à laquelle est rattachée la majorité des sites étudiés.

La sélection des sites a également répondu à un objectif de cohérence, à travers la volonté d'observer le comportement de certains types particuliers, essentiellement des signes complexes : c'est le cas des tectiformes, avec la comparaison des signes des quatre grottes de Dordogne, où ils se répartissent; des signes aviformes à Cougnac et Pech-Merle ou des signes quadrangulaires, notamment ceux présents dans les trois grottes du Monte Castillo.

Au-delà des césures géographiques ou chronologiques traditionnelles, la sélection de ces sites présente une réelle diversité dans la morphologie générale des réseaux karstiques. Ainsi, parmi les 26 grottes étudiées, se trouvent aussi bien des réseaux profonds (comme Rouffignac, plusieurs kilomètres de galeries) que courts (comme Bara-Bahau, où les gravures se trouvent rassemblées à $80 \mathrm{~m}$ de l'entrée). Autre niveau de diversité, les grottes peuvent présenter des réseaux complexes par leurs ramifications (tels que Rouffignac, Castillo ou la Pasiega) ou simples (comme les grottes couloirs des Combarelles ou de Marsoulas).

Un dernier critère de diversité concerne la régularité des surfaces pariétales. Aucune grotte ne présente des parois continuellement planes. Il reste même assez rare de rencontrer des surfaces planes importantes, comme c'est le cas à Fontanet, où des signes occupent exclusivement cet espace ou dans la grotte de la Garma (Cantabres) où un ensemble de points et de traits occupe une paroi plane longue de plusieurs mètres.

\footnotetext{
${ }^{4}$ Les autorisations d'accès à de nombreux sites constituent un élément déterminant dans la réalisation de cette analyse et l'aide apportée par les propriétaires, guides et conservateurs a été déterminante. Je tiens à remercier tous ceux qui m'ont accueilli et permis l'accès aux grottes ou galeries d'ordinaires fermées au public. L'absence d'autorisation ou les contraintes inévitables d'origines diverses rencontrées pour certains sites ont participé également à la sélection de cet échantillon et à sa restriction dans certains cas.
} 
Tableau 1

Distribution typologique des signes analysés dans l'espace franco-cantabrique

Table 1

Distribution of sign types analysed inside franco-cantabric area

\begin{tabular}{|c|c|c|c|c|c|c|c|c|c|c|c|c|c|c|c|}
\hline & $\begin{array}{l}\text { Ponctuation } \\
\text { - }\end{array}$ & $\begin{array}{l}\text { Ponctuation large } \\
\text { - }\end{array}$ & $\begin{array}{l}\text { Trait court } \\
\square\end{array}$ & Trait long & $\begin{array}{l}\text { Angulaire } \\
\wedge\end{array}$ & $\begin{array}{l}\text { Croix } \\
\times\end{array}$ & $\begin{array}{l}\text { Triangle } \\
\text { A }\end{array}$ & $\begin{array}{l}\text { Cercle } \\
\bigcirc\end{array}$ & $\begin{array}{l}\text { Quadrilatere } \\
\square\end{array}$ & Claviforme & Tectiforme & Aviforme & Ramiforme & Autres & Total \\
\hline Aldène & & & & & 1 & & & 1 & & & & & & & 2 \\
\hline Bara-Bahau & & & & & & & & & 3 & & & & & 2 & 5 \\
\hline Bédeilhac & 10 & & 1 & 8 & 2 & & & & 1 & 1 & & & & 3 & 26 \\
\hline Bernifal & 2 & 1 & 4 & 1 & 1 & 1 & & 4 & & & 11 & & 1 & 4 & 30 \\
\hline Calero II & 5 & & 1 & & & & & & & & & & & 8 & 14 \\
\hline Chufin & 10 & & 2 & 2 & 1 & & 1 & & & & & & & 1 & 17 \\
\hline Les Combarelles & & & 18 & 13 & 8 & 4 & 9 & 1 & 1 & & 6 & & & 17 & 77 \\
\hline Combe-Nègre & 3 & 3 & & & & & & & & & & & & & 6 \\
\hline Cougnac & 12 & & 7 & & & & & & & & & 8 & & 1 & 28 \\
\hline El Castillo & 14 & 13 & & 3 & 1 & 1 & & 3 & 24 & & & & 1 & 9 & 69 \\
\hline El Pendo & 3 & & 1 & & & & & & & & & & & 2 & 6 \\
\hline Font de Gaume & 3 & & 1 & 1 & & 1 & 1 & 2 & 1 & & 19 & & & 3 & 32 \\
\hline La Garma & 3 & 3 & 8 & & & & & & 1 & & & & & 1 & 16 \\
\hline La Pasiega & 5 & & & 6 & & & 2 & & 22 & & & & & 25 & 60 \\
\hline Las Aguas & 1 & & & 1 & & & & & & & & & & 3 & 5 \\
\hline Las Chimeneas & & & & & & & & & 8 & & & & & 2 & 10 \\
\hline Marsoulas & 8 & & & 4 & & & & & 2 & & & & 14 & 11 & 39 \\
\hline Mas-d'Azil & 12 & & 2 & 1 & 5 & 2 & & & & & & & & 1 & 23 \\
\hline Niaux & 2 & & & 5 & 40 & & & & & 18 & & & 6 & & 71 \\
\hline Pech-Merle & 3 & 14 & 4 & & & & 1 & 2 & & & & 1 & & 2 & 27 \\
\hline Pestillac & & & 1 & 5 & & & & & & & & & & 2 & 8 \\
\hline Le Portel & 12 & & 5 & 2 & 3 & & & & & 1 & & & & 8 & 31 \\
\hline Rouffignac & & & & 2 & 2 & & & 2 & & & 16 & & & 10 & 32 \\
\hline Santian & & & & & & 1 & & & & & & & & 16 & 17 \\
\hline Travers de Janoye & & 10 & & & & & & & & & & & & & 10 \\
\hline Villars & 23 & & 2 & 1 & & 1 & & & & & & & 1 & 3 & 31 \\
\hline Total & 131 & 44 & 57 & 55 & 64 & 11 & 14 & 15 & 63 & 20 & 52 & 9 & 23 & 134 & 692 \\
\hline
\end{tabular}


La majeure partie des surfaces décorées, et nous l'avons constaté dans l'étude du corpus, sont irrégulières (498 des 692 signes étudiés se trouvent sur des surfaces polymorphes, 93 sur des surfaces concaves). L'irrégularité de certaines surfaces rend du reste certaines d'entre elles, parfois sur toute une partie de la grotte, inappropriées à la réalisation du moindre graphisme (c'est notamment le cas de toute la galerie d'accès de la grotte de Cougnac, de celle du Travers-de-Janoye ou en outre importante des surfaces de Pestillac) ou à l'usage de certaines techniques.

Dans certains cas, comme à Rouffignac, la nature de la paroi oriente manifestement le choix de la technique. Ainsi, l'enduit pâteux sur les parois et les plafonds a facilité la réalisation de figures par l'usage des doigts, l'évolution morphologique constituant, dans ce cas précis une des « bases des contraintes imposées à l'artiste préhistorique » (Plassard, 1992 : p. 359).

Cet engobe argileux couvre des surfaces essentiellement régulières, qui ont été largement exploitées par les artistes magdaléniens qui ont fréquenté la grotte. À l'inverse, à quelques kilomètres de Rouffignac, à Bernifal, d'autres artistes ont investi prioritairement des surfaces tourmentées.

C'est, en effet, dans la zone étroite du passage que les artistes de Bernifal ont réalisé la majorité de leurs représentations (Fig. 13). L'inclinaison, l'alternance entre surfaces concaves et convexes n'ont pas empêché les graveurs d'y graver animaux (beaucoup de mammouths) et signes (tous les tectiformes de la grotte s'y concentrent, à l'exception d'un seul, qui est aussi le seul signe peint de la grotte).

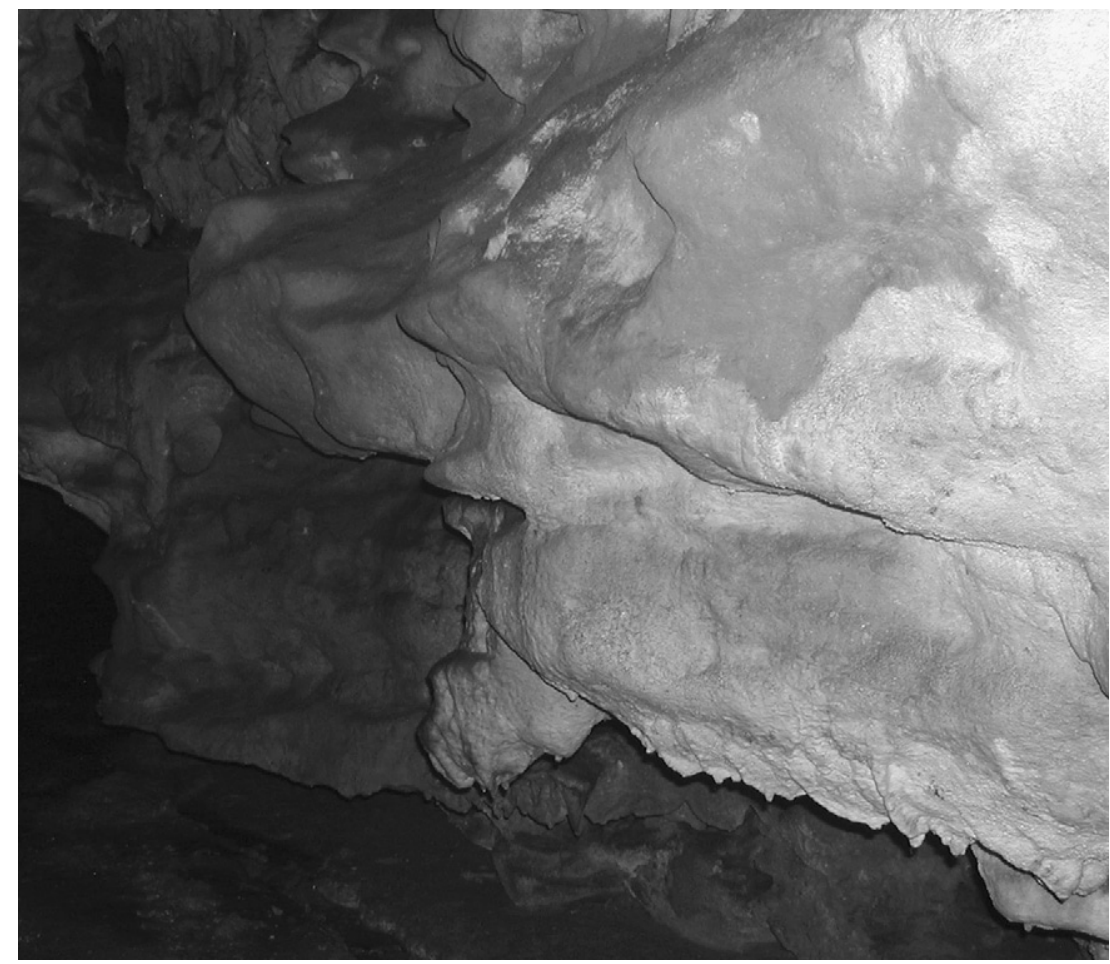

Fig. 13. Paroi gauche de la zone du Passage, Bernifal (Dordogne) (cliché Eric Robert).

Fig. 13. Left wall of "le Passage", Bernifal (Dordogne) (photography Eric Robert). 
Ainsi, malgré la difficulté supplémentaire que représentait ce secteur de la grotte par rapport à des zones plus larges et à des surfaces plus régulières, les Magdaléniens ont délibérément construit la majeure partie de leur dispositif pariétal dans cet espace étroit.

La comparaison de ces deux grottes illustre une nouvelle fois les capacités des hommes du Paléolithique supérieur à s'adapter, voire à dépasser les contraintes naturelles pour y élaborer les constructions mentales de leurs pensées.

Cela souligne d'autant plus l'intérêt à porter à la disposition de chaque représentation, dans la mesure où elle peut participer à la reconstitution du schéma de construction de la grotte.

L'ensemble des sites analysés constituait par conséquent une image qui, bien que partielle, soit assez révélatrice de la diversité des milieux souterrains auxquels ont été confrontés les hommes tous au long du Paléolithique supérieur.

La répartition typologique de cet échantillon reflète également la diversité morphologique des deux principales familles de signes, les ensembles les plus importants étant constitués des formes les plus simples (ponctuations ou traits), auxquels s'adjoignent les signes au tracé plus complexe, correspondant le plus souvent à des territoires plus définis, comme les tectiformes en Dordogne ou les quadrangulaires dans les Cantabres.

\section{La fréquence des modes d'utilisation}

La sélection des signes et des sites permet, dans sa diversité aussi bien formelle que technique, d'observer toutes les facettes et donc toutes les possibilités de combinaison avec les reliefs naturels qui avaient pu être mis en place.

L'observation de la fréquence des trois modes d'utilisation que nous avons différenciés (intégration, mise en valeur de la surface et cadrage) permet déjà de mettre en évidence des tendances particulières.

\subsection{L'intégration}

Le premier élément notable que l'on constate tient dans le nombre d'exemples extrêmement rares d'intégration du relief pour les signes. Parmi les 692 signes étudiés, seuls huit peuvent refléter une telle relation avec leur support naturel.

L'exemple qui illustre le mieux ce phénomène est un signe angulaire de la grotte du Portel, formé d'une branche peinte jointe à une crête naturelle de taille égale (Fig. 14). Ce signe se distingue d'autant plus qu'il est le seul de ce type dans la grotte du Portel, et qu'il se situe dans un renfoncement de la partie médiane de la fourche que forme le réseau karstique. Il combine une originalité au niveau de la relation avec son support, de sa nature graphique et de son emplacement dans la grotte.

Une même combinaison se remarque pour un autre exemple d'intégration : un signe quadrangulaire de la grotte du Castillo, peint en rouge. Situé à proximité du «Recoin-auxTectiformes », ce signe occupe le registre central de la paroi droite et se détache visuellement par son élaboration graphique.

Une fissure verticale le traverse, formant un axe médian autour duquel le signe se construit, sa moitié droite s'incurvant pour s'appuyer sur la limite entre paroi et plafond (Fig. 15). Si elle ne compose pas un trait à proprement parler, la fissure participe effectivement à l'aspect visuel original du signe. 


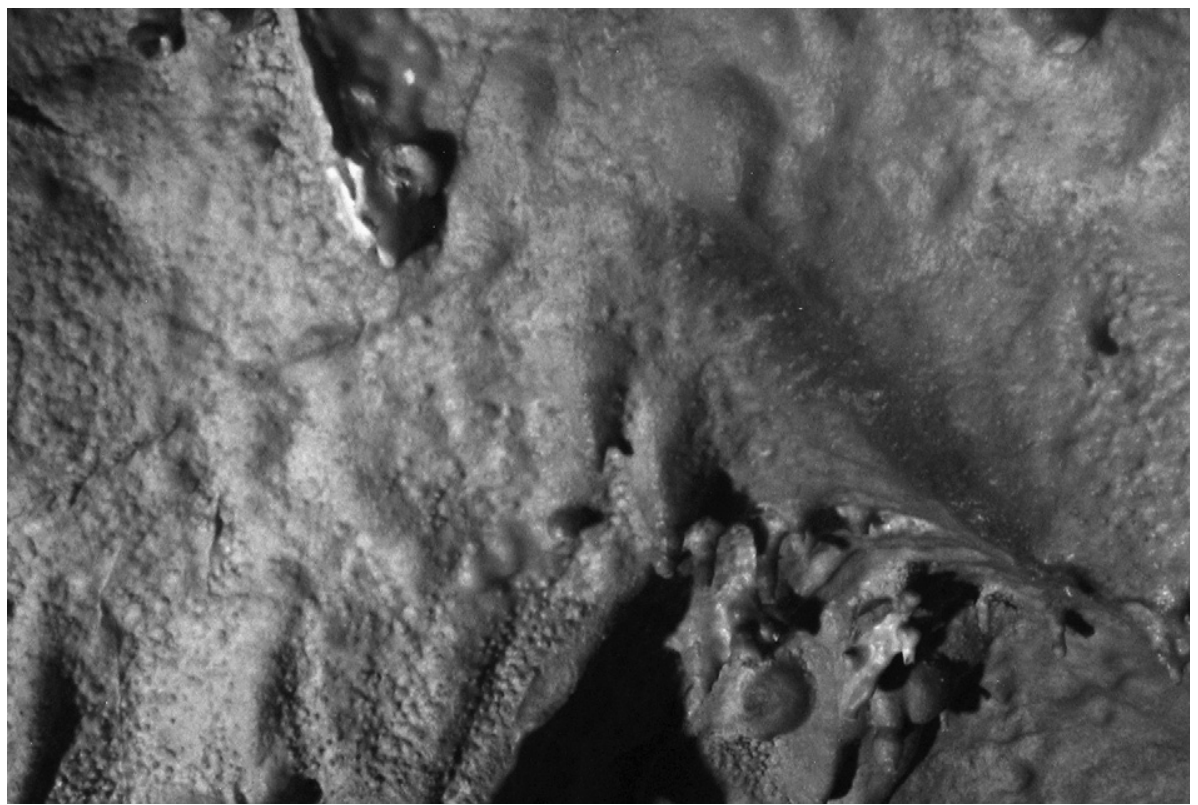

Fig. 14. Signe angulaire intégrant le relief, le Portel (Pyrénées) (cliché Eric Robert).

Fig. 14. Angular sign integrating the relief, le Portel (Pyrénées) (photography Eric Robert).

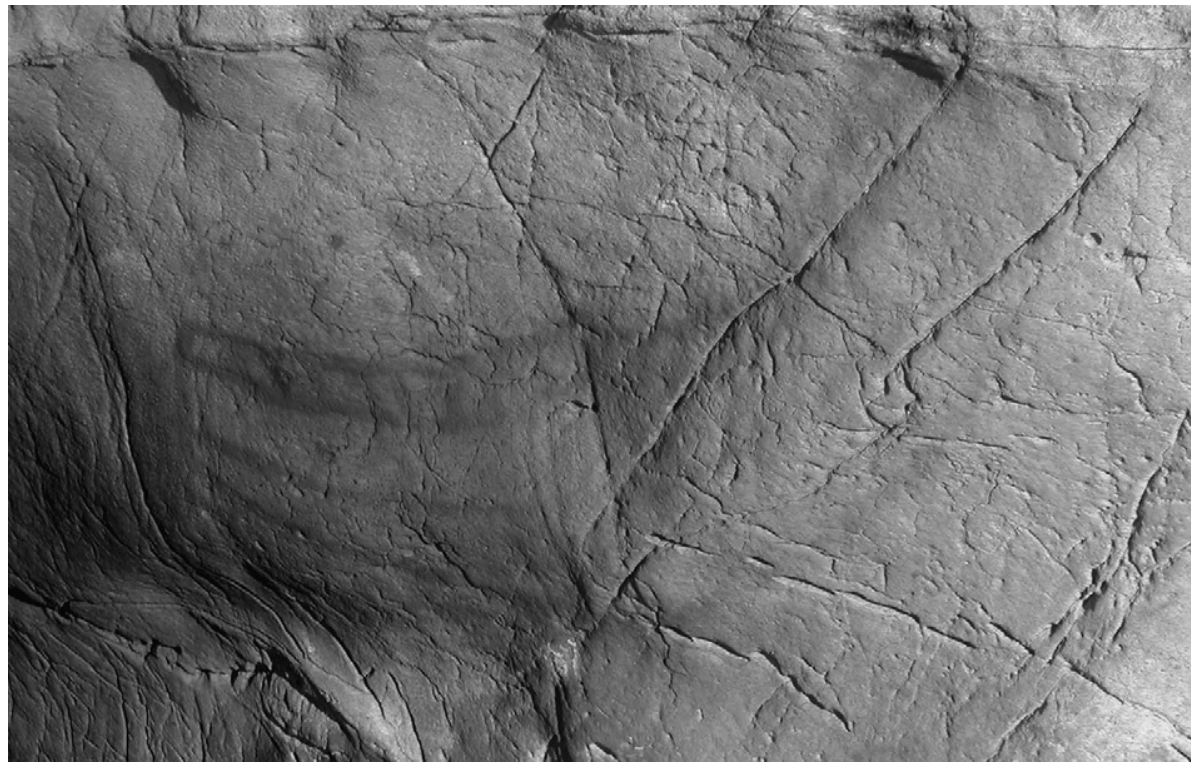

Fig. 15. Quadrangulaire rouge intégrant une fissure, El Castillo (Cantabres) (cliché Eric Robert). Fig. 15. Red quadrangular integrating a cleft, El Castillo (Cantabres) (photography Eric Robert). 
Le fait qu'il soit un des rares quadrangulaires du Castillo à posséder une excroissance participe également à sa singularisation par rapport aux autres signes du même type ; nombreux dans la grotte.

L'intégration des reliefs peut aussi se traduire par la constitution d'une image graphique originale associant plusieurs tracés avec le support.

C'est le cas dans la grotte de Font-de-Gaume, pour deux tectiformes rouges, réalisés par l'adjonction de séries de petites ponctuations. Ils sont disposés de part et d'autre d'un creux, ce dernier semblant faire lien entre ces tracés. La technique de réalisation est originale, unique à Font-de-Gaume, présente seulement pour un signe de la grotte voisine de Bernifal.

Leur emplacement, sous un auvent naturel faisant protection par rapport au passage, à l'entrée de la galerie profonde, renforce l'impression de l'individualisation d'une image combinant graphisme abstrait et relief naturel.

On constate donc une nouvelle fois une combinaison de l'originalité entre forme, utilisation du support et emplacement sur la paroi.

L'intégration des reliefs naturels apparaît au final comme une exception pour les manifestations abstraites, alors que ce mode d'utilisation est fréquemment évoqué pour les animaux.

Sans données précises, on ne peut quantifier cette différence entre ces deux familles de représentations mais elle est incontestablement révélatrice d'un traitement graphique différent, de démarches distinctes dans la réalisation des différents thèmes.

\subsection{La recherche des volumes}

Les deux autres modes d'utilisation sont apparus plus fréquemment à la lecture des signes analysés. Les reliefs utilisés sont de natures diverses et sont relativement variés. La complexité des morphologies pariétales explique en partie cette variété. Sans détailler chaque forme, on peut essentiellement distinguer les reliefs par la conjugaison de trois facteurs : volume (concave ou convexe), longueur/largeur, profondeur.

Ainsi on distingue, outre les creux des bosses, les fissures ou sillons (concaves dans les deux cas mais de largeur et de longueur plus importante pour les sillons); ainsi que les reliefs allongés étroits comme les crêtes ou les saillies, certains marquant une rupture d'orientation, telles que les arêtes.

Une dernière catégorie de reliefs est un peu particulière, celle des « bords » matérialisant ainsi les signes (ou d'autres tracés) situés en limite de parois, au niveau où la rupture est définitive.

Ces différents reliefs pariétaux illustrent la variété morphologique pariétale à laquelle ont été confrontés les hommes du Paléolithique supérieur et qu'ils ont diversement exploités.

En effet, les volumes et le cadrage ne se rencontrent pas dans les mêmes proportions, les reliefs utilisés et les signes concernés présentant également des distinctions.

La recherche des volumes n'apparaît pas comme un élément dominant dans la réalisation graphique, surtout s'il ne s'accompagne pas d'une recherche de cadrage. Cinquante-huit signes sur les 692 font, en effet, l'objet d'une utilisation de reliefs rocheux au niveau de la surface du signe, 78 autres signes faisant, eux, l'objet d'une double utilisation, surface et cadrage combinés, portant le total des signes disposés sur des volumes particuliers à 136.

Cette proportion de recherches des volumes plus limitée, qui n'est malgré tout pas mineure, peut se comprendre par la démarche particulière qui sous-tend cette utilisation.

La disposition sur des surfaces concaves ou convexes apporte, en effet, que rarement une dimension volumétrique aux signes, alors qu'elle peut être plus évocatrice pour les animaux. La 


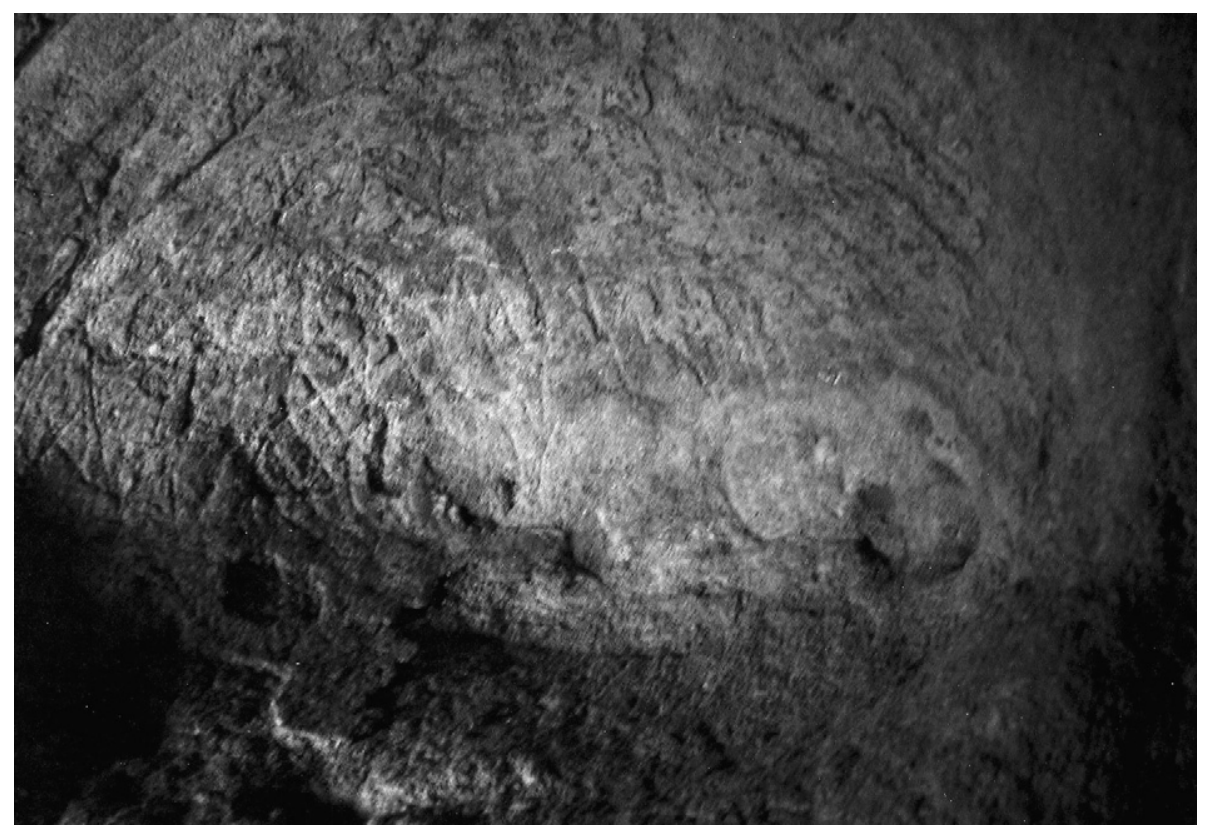

Fig. 16. Cercles gravés dans un creux, Font-de-Gaume (Dordogne) (cliché Eric Robert).

Fig. 16. Engraved circles inside a cavity, Font-de-Gaume (Dordogne) (photography Eric Robert).

combinaison des cercles et tracés arrondis situés dans un creux profond, dans la galerie principale de la grotte de Font-de-Gaume, où la forme du relief et celle du signe s'imbriquent étroitement pour constituer une image originale, reste plutôt une exception (Fig. 16).

En revanche, les volumes peuvent apparaître comme une manière de souligner les signes concernés. C'est particulièrement le cas pour certaines séries de signes.

L'exemple des ponctuations de Villars, dont nous avons eu l'occasion de souligner la position stratégique, est assez révélateur de ce phénomène. En effet, la plupart des ponctuations présentes tout au long du parcours souterrain se situent sur des petites bosses de la paroi, des protubérances de taille certes modeste mais qui soulignent leur position, alors même que leur situation topographique, que nous avons précédemment soulignée, laisse apparaître que ces signes occupent une place stratégique indiscutable dans la constitution du discours pariétal.

Dans un autre registre, les bandes de ponctuations de la grotte de Chufin (Cantabres) illustre la répétition locale d'une attention à un support particulier. Au niveau d'une plate-forme qui domine, paroi gauche, la salle du fond de cette grotte, se trouvent plusieurs signes formés par des séries de ponctuations alignées (Fig. 17). Elles s'inscrivent dans des concavités de la paroi et ce alors même qu'un grand segment plan de la paroi n'a pas été investi. Ces concavités soulignent, de près comme depuis la salle, ces ensembles abstraits qui dominent ce segment de la grotte mais aussi l'ensemble peint profond de la grotte de Chufin, distinct des gravures du porche d'entrée.

Les récurrences s'observent, toutefois, rarement au-delà d'un même dispositif pariétal. L'association point/bosse, qui domine à Villars, constitue l'essentiel des exemples observés dans les grottes. C'est aussi le cas pour les alignements de points associés à des concavités à Chufin.

Ces deux exemples illustrent la principale tendance observée dans la recherche répétée de certains volumes, à savoir plutôt des spécificités de sites, que des spécificités de types. Si les 


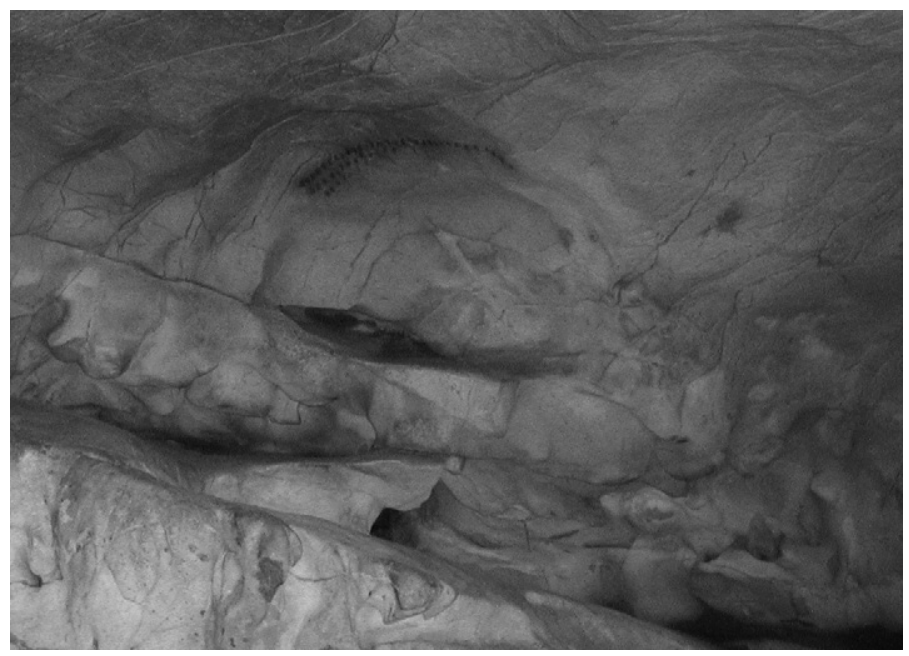

Fig. 17. Bandes de ponctuations rouge inscrites dans une concavité, vues depuis la salle du fond, Chufin (Cantabres) (cliché Eric Robert).

Fig. 17. Bands of red punctuations disposed inside a cavity, observed from final room, Chufin (Cantabres) (photography Eric Robert).

ponctuations sont de loin le type le plus fréquemment associé à des volumes particuliers, cela correspond à la particularité de certains dispositifs pariétaux.

\subsection{Les cadrages}

Les exemples de cadrages constituent la majeure partie des cas que nous avons observés. Pas moins de 223 signes de notre inventaire font, en effet, l'objet de ce mode d'utilisation, auxquels il faut ajouter les 83 cas de signes faisant aussi l'objet d'une recherche de volumes, voire d'intégration. L'ensemble des 306 cas représente $44 \%$ des signes de notre corpus (Tableau 2).

La très grande majorité des cadrages sont partiels. Seuls 16 signes font l'objet d'un cadrage entier, c'est-à-dire, que l'ensemble du contour du signe est délimité par des reliefs naturels. Ce sont généralement des signes situés dans des surfaces concaves, favorisant ainsi une délimitation complète qui peut associer plusieurs reliefs différents.

Un groupe de 80 ponctuations de la Pasiega B illustre ce phénomène. Les points sont intégrés entre plusieurs fissures et le rebord supérieur de la paroi (Fig. 18) qui forment le cadre-limite du signe.

Cette faible proportion ne doit pas limiter l'importance indéniable des éléments de reliefs pour le cadrage qui participent à la mise en place du décor sur la paroi. L'emplacement n'est plus neutre, surtout lorsque les figures voient leur forme, même, influencée par les particularités du support.

Lorsque l'on observe la fréquence des exemples de cadrage par type de signe, peu se distinguent beaucoup de la moyenne. Ainsi, des types élaborés, comme les tectiformes ou les quadrangulaires, malgré leur originalité et leur statut de « marqueurs ethniques », ne témoignent pas, dans leur ensemble, d'une disposition plus fréquente sur des reliefs particuliers.

Si toutefois, on observe indépendamment les dispositifs pariétaux, on note des fréquences alors bien différentes. Ainsi, les six tectiformes des Combarelles font tous l'objet d'un cadrage 
Tableau 2

Distribution des cas de cadrage des signes par type

Table 2

Repartition of frame examples by types of sign

\begin{tabular}{llllllll}
\hline Type de signes & Ponctuation & Ponctuation large & Trait & Trait Angulaire Croix Triangle Cercle Quadrilatère Claviforme Tectiforme Aviforme Ramiforme Autres Total
\end{tabular} court long

\begin{tabular}{lrrrrrrrrrrrrrrrr}
\hline Exemples de cadrage/type & 51 & 30 & 19 & 20 & 16 & 4 & 5 & 11 & 32 & 15 & 25 & 4 & 12 & 62 & $\mathbf{3 0 6}$ \\
Effectif total/type & 131 & 44 & 57 & 55 & 64 & 11 & 14 & 15 & 63 & 20 & 52 & 9 & 23 & 134 & $\mathbf{6 9 2}$ \\
$\begin{array}{l}\text { Pourcentage de cadrage } \\
\text { par type de signes (\%) }\end{array}$ & 39 & $\mathbf{6 8}$ & 33 & 40 & 25 & 36 & 36 & $\mathbf{7 3}$ & 51 & 75 & 48 & 44 & 52 & 46 & $\mathbf{4 4}$ \\
\hline
\end{tabular}




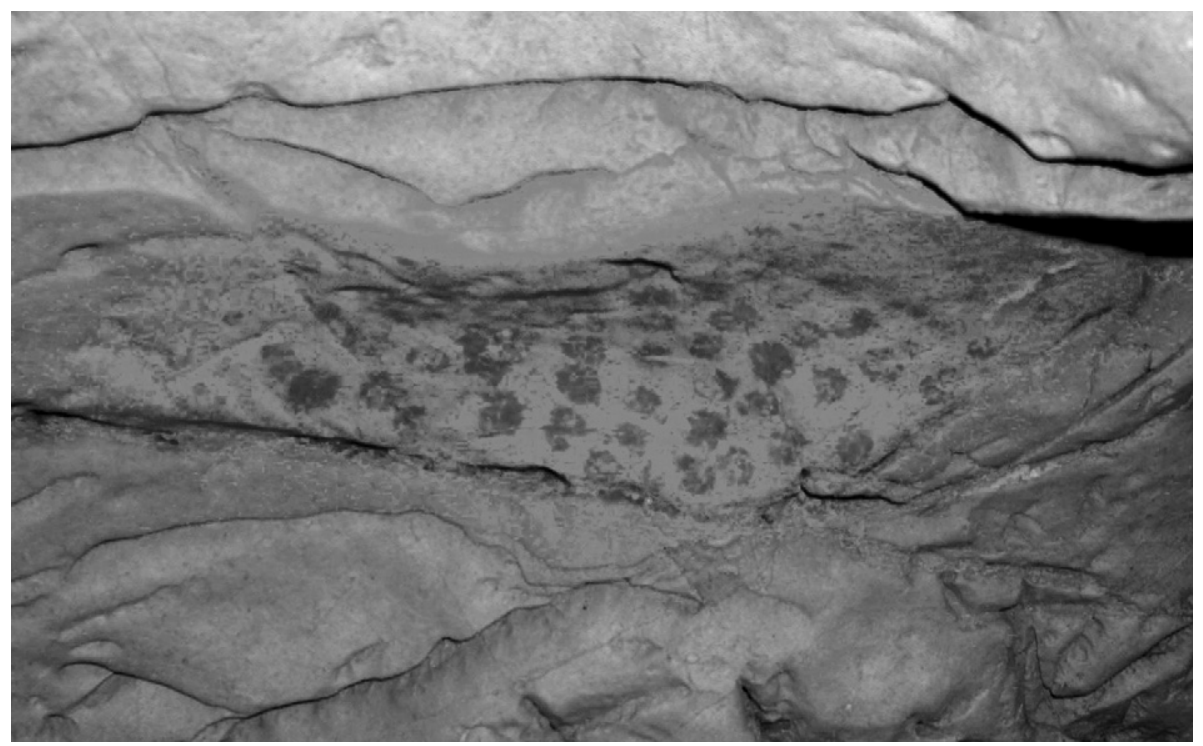

Fig. 18. Groupe de ponctuations rouge-inscrite entre des fissures, la Pasiega B (Cantabres) (cliché Eric Robert).

Fig. 18. Group of red punctuations disposed between clefts, la Pasiega B (Cantabres) (photography Eric Robert).

particulier, en l'occurrence, ils s'appuient tous sur le bord inférieur de la paroi. À l'inverse, seuls trois des seize tectiformes de Rouffignac sont cadrés par des reliefs naturels.

Cette différence des tectiformes d'une grotte à l'autre, que l'on retrouve sur d'autres plans (topographique, technique ou dans les liaisons graphiques), confirme que les rapports entre les signes et leurs supports ne sont pas uniformes mais qu'ils participent, comme les autres niveaux de lecture, à la distinction des « pensées et croyances de leurs créateurs » (Vialou, 1987 : p. 316).

Les signes à structure simple, comme les ponctuations digitées ou les traits sont moins concernés que la moyenne des signes. Pour les ponctuations, c'est surtout vrai lorsqu'elles sont isolées. Leur morphologie, leur taille plus modeste, peuvent être à l'origine de cette moindre proportion qui pourrait aussi être révélatrice d'un rôle graphique différent où leur mise en valeur par le cadrage ne constitue pas la priorité.

Deux types de signes, outre les claviformes de notre sélection qui s'avèrent être presque exclusivement ceux de Niaux, se distinguent particulièrement dans la proportion de leur mise en valeur, mettant en lumière des phénomènes différents : les cercles (73\% de signes cadrés par rapport aux reliefs) et les ponctuations larges (68\%).

Les premiers ne constituent qu'un faible corpus (11 des 15 signes classés dans ce type), reflétant des exemples, techniques et morphologiques, très différents ; on y retrouve, aussi bien, les signes ovalaires de la zone du Passage-de-Bernifal, le signe dans le creux de Font-de-Gaume ou un cercle échancré rouge de Pech-Merle. Toutefois, dans chacun de ces contextes distincts, ces signes que l'on ne peut rapprocher que difficilement, occupent une place tout à fait particulière. Les cercles échancrés sont une forme originale aux grottes quercynoises, Pech-Merle et Roucadour étant les deux seules grottes à en posséder, alors que les signes ovalaires du Castillo tendent eux aussi à s'agréger dans un même espace. Le signe de Font-de-Gaume, enfin lui, reste assez exceptionnel par sa forme comme par son support naturel prononcé. 


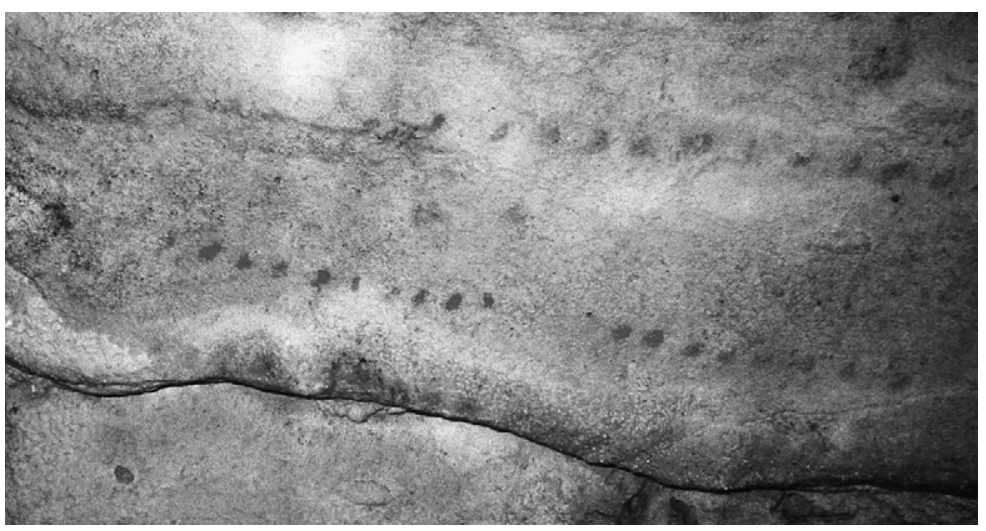

Fig. 19. Alignements horizontaux de points rouges, le Travers de Janoye (Quercy) (cliché Pierre Cassan et Eric Robert). Fig. 19. Horizontal lines of red points, le Travers de Janoye (Quercy) (photography Pierre Cassan and Eric Robert).

\subsection{Le cas des ponctuations larges}

Ce sous-type particulier de la famille des ponctuations révèle-lui une situation plus complexe. Les signes en relation avec les reliefs sont plus particulièrement des groupes de ponctuations larges (alignements, nappes...), pour lesquels on observe une indiscutable tendance à se construire et à s'orienter autour d'accidents naturels. Ce phénomène est particulièrement sensible dans les grottes quercynoises, le Travers-de-Janoye en constituant le symbole.

En effet, tous les alignements de points situés dans la seule galerie décorée sont tous construits en fonction de reliefs pariétaux, à chaque fois différente. Cette association récurrente se matérialise dans la forme même des signes et plus particulièrement de leur orientation. Chacun des alignements de points présente une situation distincte :

- un alignement oblique de cinq points le long d'une fissure ;

- quatre alignements horizontaux de points superposés, parallèles à des crêtes et au rebord de la paroi (Fig. 19) ;

- deux alignements obliques parallèles, qui « suivent très exactement une concavité transversale de la paroi, large de 0,17 à $0,18 \mathrm{~m}$, puis, quand cette concavité disparaît, [...] partent à l'horizontale jusqu'à l'orée de la fissure » (Clottes et Lautier, $1981:$ p. 109) ;

- un alignement vertical de 13 points qui suit le rebord de la paroi (Fig. 20).

Ce dernier signe est l'exemple le plus évocateur de cette systématisation dans l'organisation graphique de la grotte. Les ponctuations larges dominent le corpus, marquant par leur lien étroit avec le support une construction particulière, caractère dominant du dispositif pariétal du Travers de Janoye.

Une tendance similaire s'observe dans deux autres sites quercynois. À Pech-Merle, les groupes de ponctuations larges sont aussi nombreux dans les différentes galeries. Sur le panneau des chevaux ou avec les femmes-bisons mais surtout dans la galerie du Combel, on retrouve l'influence des reliefs naturels sur l'organisation de ces groupements de ponctuations. C'est notamment le cas d'un signe en grille « qui se loge entre les draperies convergentes et encadre un pointement calcaire » (Lorblanchet, $1989:$ p. 86). 


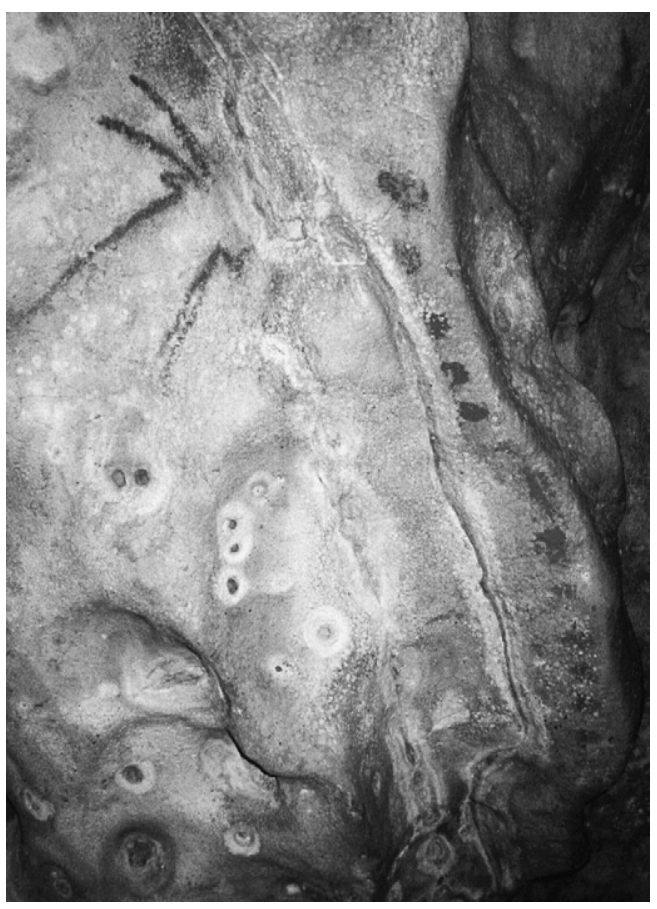

Fig. 20. Alignement vertical de points rouges, le Travers de Janoye (Quercy) (cliché Pierre Cassan et Eric Robert). Fig. 20. Vertical line of red points, "le Travers de Janoye" (Quercy) (photography Pierre Cassan and Eric Robert).

Dans le réseau supérieur de la grotte de Combe-Nègre, découverte en 2001, les ponctuations représentent l'unique thème présent sur les parois. Agencées en lignes ou en groupes, elles témoignent, comme au Travers-de-Janoye, de l'influence de leur support.

En l'espace de quelques mètres, elles s'organisent autour de plusieurs reliefs, entre autres une « nappe de grosses ponctuations noires s'enroulant autour d'un pendant rocheux triangulaire » (Jaubert, 2001 : p. 196).

La similarité entre ces différentes galeries est assez étonnante. Même si les reliefs ne sont pas identiques, la forme particulière de ces signes et leur lien étroit avec leurs supports leur confère une spécificité toute particulière. Cette spécificité est d'autant plus intéressante qu'elle s'observe dans des ensembles graphiques tous attribués au Gravettien.

Cette spécificité se confirme dans d'autres grottes quercynoises. Cougnac possède, en effet, une série de points de ce type dans un boyau situé au pied des panneaux de la rotonde principale. La fin du dispositif orné de la Grotte-des-Merveilles, dans la moitié Nord du Lot, est ornée de 13 grosses ponctuations rouges sur « un bec rocheux fissuré et concrétionné » (Lorblanchet, 1984 : p. 493), dont plusieurs s'inscrivent dans des creux du pilier.

Dans la grotte plus septentrionale du Moulin-de-Laguenay (Corrèze), une série de ponctuations, réparties au plafond, semble faire l'objet d'une « utilisation préférentielle des creux du support » (Pigeaud et Primault, 2006 : p. 38), qui n'est pas sans rappeler les points du plafond du Combel.

La systématisation de la construction de ces signes par rapport à la paroi pourrait donc apparaître comme caractéristique d'un espace culturel, en l'occurrence le Gravettien quercynois. 
Toutefois, la présence des exemples similaires d'utilisations dans des contextes culturels différents amène à pondérer cette conclusion.

Ainsi, deux signes situés dans les grottes magdaléniennes de Font-de-Gaume et de Bernifal, font, aussi, l'objet d'une disposition particulière. La situation des nappes de « points-main » de Chauvet (Ardèche) ou de la Grotte-aux-Points (Gard) voisine, renforce l'idée que l'on se trouve face à une constance dans la construction de ce type, tout au long du Paléolithique supérieur.

Les groupes de ponctuations larges se distinguent manifestement par leur étroit rapport à la paroi, la forme (orientation ou construction) de ces alignements de points étant indubitablement influencée par la morphologie de leur support naturel.

La position souvent à l'écart de ces signes, rarement associés à d'autres thèmes (les mains au Castillo, les bouquetins au Travers-de-Janoye, les chevaux du panneau de Pech-Merle), conduit à s'interroger sur la place que ces signes pouvaient occuper au sein des dispositifs pariétaux. Objets d'un investissement particulier dans leur réalisation graphique, ils se distinguent des autres familles de signes au sein desquelles on ne retrouve jamais, au-delà d'un site, une telle constance.

\section{L'influence des reliefs naturels sur le tracé}

L'exemple des ponctuations témoigne du lien étroit que peuvent entretenir les signes et leurs supports naturels, jusqu'à influencer la forme même du signe. Si cette influence peut être difficile à identifier étant donné qu'à la différence des animaux, on ne connaît pas la forme typique d'un signe, elle se manifeste tout de même clairement à de nombreuses reprises.

C'est le cas pour les exemples d'intégration de reliefs, où le tracé du signe est directement lié au support. C'est le cas également de la situation particulière de certains signes, comme les cercles et arrondis gravés de Font-de-Gaume ou comme plusieurs groupes de ponctuations inscrits dans des concavités à Chufin. L'ensemble des alignements de ponctuations larges, enfin, témoigne de cette influence dans leur orientation.

Au-delà de la composition, de la délimitation ou de l'orientation, l'utilisation des reliefs naturels peut conduire à une interaction avec la forme même des tracés.

Deux exemples illustrent la portée de ce phénomène, d'autant plus intéressants qu'ils se situent aux deux extrémités du Paléolithique supérieur.

Le premier d'entre eux provient de la grotte de l'Aldène (Hérault), dont une étude récente a permis de fixer la chronologie (Ambert et al., 2005). Une datation de la fermeture du proche a, en effet, pu être obtenue et de fixer le passage des hommes entre 37000 et 24000 BP. La datation d'un charbon de bois, prélevé dans une couche de la galerie ornée, à $30260 \pm 220$ BP, associée à des recoupements avec l'art de Chauvet, permet de fixer les représentations gravées de l'Aldène à l'Aurignacien.

Le signe est classé dans la famille des cercles. Il est constitué de plusieurs séries de traits incurvés, réalisés successivement, sur l'arrière-train d'un félin, qu'il précède dans la réalisation. Inspiré des griffades d'ours de la paroi, par sa composition issue « pour partie d'une griffade et pour l'autre de tracés imitant l'œuvre du plantigrade » (Vialou, 1984 : p. 17), il est cadré sur plusieurs plans par un ressaut de la paroi et par plusieurs fissures (Fig. 21). L'influence est ici double technique (voire symbolique ?) par rapport aux griffades, morphologique par rapport au cadre : « il n'est pas interdit de penser que ces limites ont déterminé la dimension du signe » (Vialou, 1979 : p. 37).

L'influence du cadre est aussi perceptible pour le second exemple. Situé dans la grotte de Pestillac (Lot), il appartient à un ensemble magdalénien, qui pourrait être rattaché au Magdalénien supérieur, de par la présence de figures féminines stylisées (Sentis, 2000). 


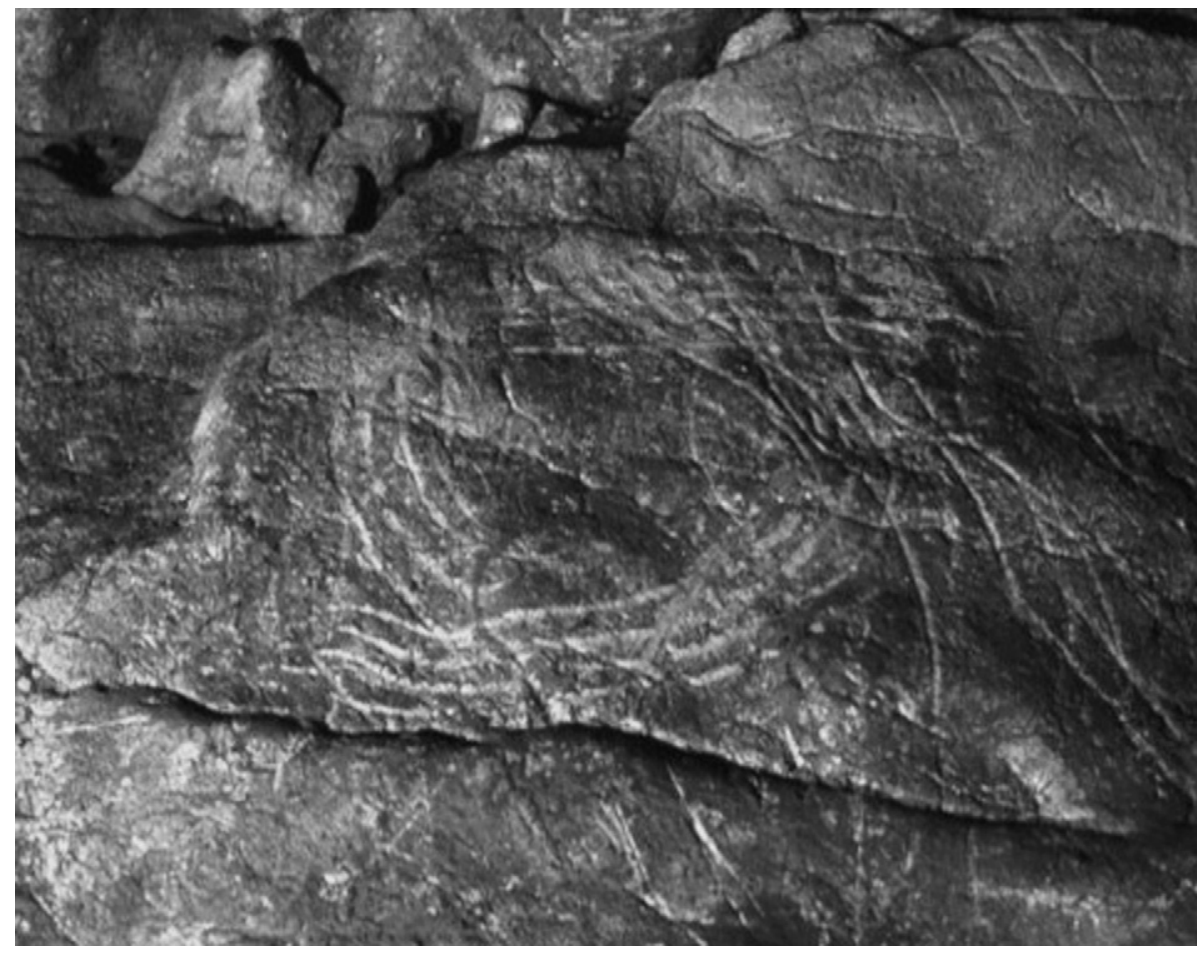

Fig. 21. Cercle gravé, l'Aldène (Hérault) (cliché Denis Vialou).

Fig. 21. Engraved circle, l’Aldène (Hérault) (photography Denis Vialou).

Ce signe est formé de douze lignes parallèles incurvées, certaines d'entre elles étant peut-être munies d'une boucle latérale, formant des claviformes possibles au milieu de la série.

Elles sont hautes d'environ $18 \mathrm{~cm}$ pour les premières situées à gauche, de $11 \mathrm{~cm}$ pour celles situées à droite. «Cette différence [...] est dictée par la morphologie de la paroi car l'artiste a utilisé toute la surface d'un panneau propice à la gravure » (Sentis, 2000 : p. 141).

Combinée à l'écartement régulier entre les lignes, cette inscription dans le support pariétal de ce signe est assez remarquable. L'homogénéité générale de ce signe, qui prend forme dans le support, conduit à faire de ces 12 lignes parallèles et malgré la présence de boucles sur certaines d'entre elles, une entité graphique à part entière (Fig. 22).

Cet exemple révèle aussi la volonté, par l'artiste, de l'inscription dans le support de son œuvre graphique. Il y a un choix manifeste de suivre les limites du support et non de respecter une homogénéité dans la hauteur des lignes. Le relief n'agit pas ici comme une contrainte mais plutôt comme un guide, sauf à considérer que l'artiste aurait automatiquement réalisé les tracés de gauche à droite en « découvrant » progressivement la forme du support.

Les deux exemples de l'Aldène et de Pestillac illustrent, de manière différente, mais complémentaire, la part indéniable jouée par les supports naturels, non seulement dans la disposition des signes mais jusque dans leur forme même.

Ils confirment l'élaboration par les hommes du Paléolithique supérieur d'un véritable discours pariétal, où le contexte karstologique constitue la trame de l'expression graphique. Le support naturel apparaît ici dans sa vraie nature de support de la pensée. 


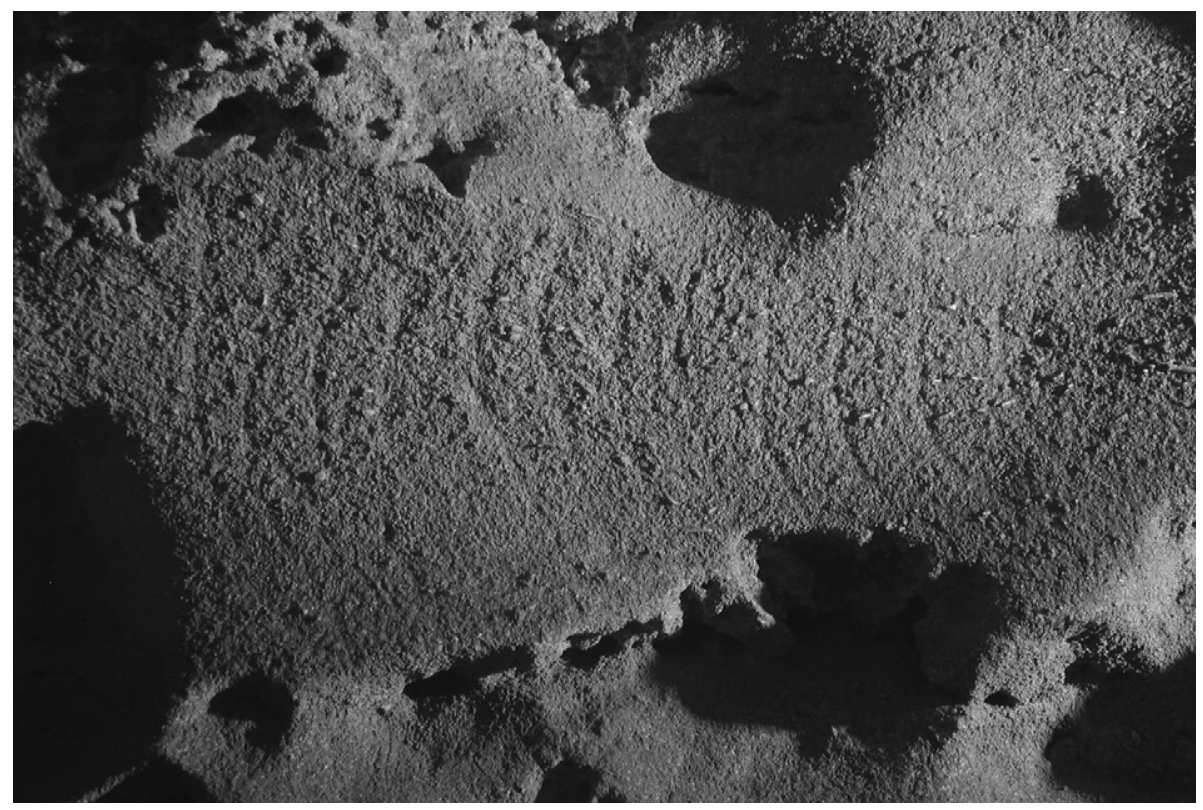

Fig. 22. Signe formé de 12 barres parallèles incurvées, Pestillac (Quercy) (cliché Eric Robert).

Fig. 22. Sign composed by 12 incurvated parallel lines, Pestillac (Quercy) (photography Eric Robert).

\section{Conclusion}

L'analyse des relations entre les signes et leurs supports confirme que la place accordée par les artistes à ces tracés abstraits n'est pas secondaire.

Près de $60 \%$ des signes que nous avons observés dans les grottes du Paléolithique supérieur européen font l'objet d'une disposition particulière sur les parois, le plus souvent destinée au cadrage, mais aussi pour une recherche de volumes.

Toutes les formes abstraites sont concernées, on n'observe aucune exclusion technique, ni morphologique dans l'utilisation des supports.

$\mathrm{Si}$, à la différence des animaux, les signes ne font qu'exceptionnellement l'objet d'une intégration des reliefs rocheux, ils entretiennent des liens étroits avec leurs supports pariétaux, liens qui peuvent se traduire par une influence réelle sur leur morphologie. La maîtrise graphique manifestée par les auteurs dans l'utilisation des reliefs révèle une adaptation totale à ce milieu contraignant que sont les réseaux karstiques.

La diversité des exemples observés au cœur des trois modes d'utilisation du relief ne fait pas émerger un modèle de construction uniforme, mais révèle plutôt une grande liberté de création graphique. Cette liberté se traduit, y compris, pour des ensembles d'apparence homogène, voire monotone, comme les signes tectiformes de la région des Eyzies.

La lecture croisée du support avec d'autres critères d'analyse, du contexte naturel mais aussi technique ou graphique, semble révéler des interrelations, comme pour les points de Villars, révélatrices de la constitution de messages graphiques.

À travers la combinaison de ces angles d'observation et la diversité des situations observées au cœur des dispositifs pariétaux, nous sommes conduits à nous interroger sur le statut des signes. 
Des convergences apparaissent autour de signes différents, alors que des types homogènes présentent, d'une grotte à l'autre, des modalités d'élaborations distinctes.

Alors que l'observation du rapport des signes avec leurs supports nous éclaire sur la richesse et la complexité des choix graphiques des artistes, la prise en compte de tous les contextes des signes, au-delà de l'ensemble des représentations préhistoriques, doit nous permettre de dépasser la reconstitution des manières de faire des artistes, pour approcher une nouvelle dimension de la pensée humaine, les «manières de dire » des hommes de la Préhistoire.

\section{Références}

Ambert, P., Guendon, J.-L., Galant, P., Quinif, Y., Grunesein, A., Colomer, A., et al., 2005. Attribution des gravures paléolithiques de la grotte d'Aldène (Cesseras, Hérault) à l'Aurignacien par la datation des remplissages géologiques. Compte Rendu Palevol 4, 275-284.

Capitan, L., Breuil, H., 1901. Une nouvelle grotte avec figures peintes sur les parois à l'époque paléolithique. Comptes rendus des Séances de l'Académie des Sciences 493-495.

Casado López, M.P., 1977. Los signos en el arte paleolítico de la península ibérica. Monografías arqueológicas, 20, Zaragoza, 1-327.

Clottes, J., Lautier, J., Rouzaud F., 1981. La grotte ornée paléolithique du Travers-de-Janoye, à Penne.In: Congrès Préhistorique de France. $21^{\mathrm{e}}$ session, Montahau-Cahors. pp. 105-115.

Delluc, B., Delluc, G., 1974. La grotte de Villars (Dordogne). Gallia Préhistoire 17, 1-67.

Delluc, B., Delluc, G., Lejeune, M., Soleihavoup, F., 1986. Lecture analytique des supports rocheux et relevé synthétique. L'Anthropologie 90, 801-804.

Gonzalez, R., 2001. Art et espace dans les grottes paléolithiques cantabriques. Éditions Jérôme Million, Grenoble.

Jaubert, J., 2001. Un nouveau sanctuaire paléolithique en Quercy : les grottes « ornées » de Combe-Nègre à Frayssinet-leGélat (Lot). Bulletin de Préhistoire du Sud-Ouest 8, 195-196.

Laming-Emperaire, A., 1962. La signification de l'art rupestre paléolithique. Éditions Picard, Paris.

Lejeune, M., 1985. La paroi des grottes, premier « mur » support artistique et document archeologique. Le mur dans l'art et l'archéologie. Art et Fact 2, Liège 15-24.

Leroi-Gourhan, A., 1958a. La fonction des signes dans les sanctuaires préhistoriques. Bulletin de la Société Préhistorique Française 55, 307-321.

Leroi-Gourhan, A., 1958b. Le symbolisme des grands signes dans l'art pariétal paléolithique. Bulletin de la Société Préhistorique Française 55, 384-398.

Leroi-Gourhan, A., 1966. Les religions de la préhistoire. Éditions PUF 1966, réédition 1986, Paris.

Leroi-Gourhan, A., 1972. Résumé des cours 1971-1972. Annales du Collège de France 407-419.

Lorblanchet, M., 1984. Grotte-des-Merveilles. In : L'art des cavernes. Atlas des grottes ornées de la Préhistoire, Paris, Imprimerie nationale, Ministère de la Culture, pp. 490-494.

Lorblanchet, M., 1989. Nouvelles découvertes d'art pariétal paléolithique de Quercy. In : L'art pariétal paléolithique. Actes des Colloques de la direction du Patrimoine, Périgueux-Le Thot, novembre 1984, pp. 79-105.

Lorblanchet, M., 1993. Le support. In : Groupe de réflexion sur l'art pariétal paléolithique, l'art pariétal paléolithique, techniques et méthodes d'étude. Éditions CTHS, Paris, pp. 69-80.

Lorblanchet, M., Delpech F., Renault P., Andrieux C., 1973. La grotte de Sainte-Eulalie à Espagneac (Lot). Gallia Préhistoire 161, 3-62.

Otte, M., 1997. Constitution d'une grammaire plastique préhistorique. L'Anthropologie 101, 5-23.

Pales, L., St Pereuse M., 1969. Les gravures de la marche mémoire 7, tome 1 « Félins et l'ours ». Publication de l'Institut de Préhistoire de l'Université de Bordeaux, Delmas, Bordeaux.

Pigeaud, R., 1998. Comment placer une figure sur un volume : les choix de l'artiste magdalénien. Exemples de comportements, appliqué à la représentation du cheval. Mémoire de DEA, Muséum National d'Histoire Naturelle.

Pigeaud, R., Primault, J., 2006. La grotte ornée du Moulin-de-Laguenay (Lissac-sur-Couze, Corrèze). Bulletin de la Préhistoire du Sud-Ouest 13/1, 37-48.

Plassard, J., 1992. Réflexion sur 1'art de Rouffignac. L'Anthropologie 96, 359-362.

Raphaël, M., 1986. Trois essais sur la signification et l'art paléolithique. Le couteau dans la plaie, Kronos, Paris.

Robert, E., 2006. Les signes et leurs supports pariétaux. Analyse comparée des rapports entre les représentations abstraites et les reliefs naturels dans les grottes ornées du Paléolithique supérieure au sein de l'espace franco-cantabrique. Mémoire de thèse de l'Université de Paris 1 Panthéon Sorbonne. 
Sanz de Santuola, 1880. Breves apuntes sobre algunos objetos prehistóricos de la provincia de Santander. Imp. Telesforo Martinez, Blanca.

Sauvet, G., Tosello, G., 1998, Le mythe paléolithique de la caverne. In : Sacco, F., Sauvet, G. (Éds.), Le propre de l'homme, Psychanalyse et préhistoire. Delachaux et Niestlé, coll. Champs psychanalytiques, 1998, Paris, pp. 55-90.

Sauvet, G., 1993. Les signes préhistoriques. In : Groupe de réflexion sur l'art pariétal paléolithique, l'art pariétal paléolithique, techniques et méthodes d'étude. Éditions CTHS, Paris, pp. 219-234.

Sauvet, G., Sauvet, S., Wlodarczyk, A., 1977. Essai de sémiologie préhistoirique (pour une théorie des premiers signes graphiqueq de l'homme). Bulletin de la Société Préhistorique Française 4, 545-558.

Sentis, J., 2000. Les gravures de la grotte de Pestillac (Montcabrier, Lot). Bulletin de la Préhistoire du Sud-Ouest 7, 133-143.

Ucko, P., Rosenfeld, A., 1966. L'art paléolithique. Éditions Hachette, Univers de connaissance, Paris.

Vialou, D., 1979. La grotte de l'Aldène à Cesseras (Hérault). Gallia Préhistoire 22, 1-85.

Vialou, D., 1984. Grotte d'Aldène. In : L'Art Paléolithique de la France méditerranéenne. Musée de Carcassonne (31 octobre - 30 novembre 1984), pp. 17-18.

Vialou, D., 1986. L'art des grottes en Ariège Magdalénienne. $22^{\mathrm{e}}$ supplément à Gallia Préhistoire. Éditions du CNRS, Paris.

Vialou, D., 1987. D'un tectiforme à l'autre. In : Bulletin de la Société Historique et Archéologique du Périgord. Actes du $39^{\mathrm{e}}$ congrès d'études régionales tenu à Sarlat les 26 et 27 avril 1986, pp. 307-317.

Vialou, D., 2004a. Architecture de l'art pariétal paléolithique. In : Lejeune, M. (Ed.), L'art pariétal paléolithique dans son contexte naturel. Actes du colloque 8.2, Congrès de l'UISPP, Liège, 2-8 septembre 2001, Liège, ERAUL 107, pp. 7-14.

Vialou, D., (dir.), 2004b. La préhistoire, Histoire et Dictionnaire. Éditions Robert Laffont, collection Bouquins, Paris. 